\title{
Topological equivalence between two classes of three- dimensional steady cavity flows: a numerical-experimental analysis
}

\author{
Citation for published version (APA): \\ Contreras Osorio, S., Ataei-Dadavi, I., Speetjens, M., Kleijn, C., Tummers, M. J., \& Clercx, H. (2019). \\ Topological equivalence between two classes of three-dimensional steady cavity flows: a numerical- \\ experimental analysis. Physics of Fluids, 31(12), 1-16. [123601]. https://doi.org/10.1063/1.5126497
}

DOI:

10.1063/1.5126497

Document status and date:

Published: 01/12/2019

\section{Document Version:}

Publisher's PDF, also known as Version of Record (includes final page, issue and volume numbers)

\section{Please check the document version of this publication:}

- A submitted manuscript is the version of the article upon submission and before peer-review. There can be important differences between the submitted version and the official published version of record. People interested in the research are advised to contact the author for the final version of the publication, or visit the $\mathrm{DOI}$ to the publisher's website.

- The final author version and the galley proof are versions of the publication after peer review.

- The final published version features the final layout of the paper including the volume, issue and page numbers.

Link to publication

\footnotetext{
General rights

- You may freely distribute the URL identifying the publication in the public portal. follow below link for the End User Agreement:

www.tue.nl/taverne

Take down policy

If you believe that this document breaches copyright please contact us at:

openaccess@tue.nl

providing details and we will investigate your claim.
}

Copyright and moral rights for the publications made accessible in the public portal are retained by the authors and/or other copyright owners and it is a condition of accessing publications that users recognise and abide by the legal requirements associated with these rights.

- Users may download and print one copy of any publication from the public portal for the purpose of private study or research.

- You may not further distribute the material or use it for any profit-making activity or commercial gain

If the publication is distributed under the terms of Article $25 \mathrm{fa}$ of the Dutch Copyright Act, indicated by the "Taverne" license above, please 


\section{Topological equivalence between two classes of three-dimensional steady cavity flows: A numerical-experimental analysis ${ }^{\circledR}$}

Cite as: Phys. Fluids 31, 123601 (2019); https://doi.org/10.1063/1.5126497

Submitted: 03 September 2019 . Accepted: 19 November 2019 . Published Online: 06 December 2019

P. S. Contreras (D, I. Ataei-Dadavi, M. F. M. Speetjens, C. R. Kleijn (D, M. J. Tummers, and H. J. H. Clercx

\section{COLLECTIONS}

EP This paper was selected as an Editor's Pick

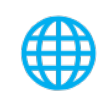

View Online

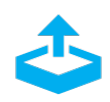

Export Citation

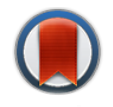

CrossMark

\section{ARTICLES YOU MAY BE INTERESTED IN}

Oscillatory flow of Maxwell fluid in a tube of isosceles right triangular cross section

Physics of Fluids 31, 123101 (2019); https://doi.org/10.1063/1.5128764

Effect of porosity on the settling behavior of a 2D elliptic particle in a narrow vessel: $A$ lattice-Boltzmann simulation

Physics of Fluids 31, 123301 (2019); https://doi.org/10.1063/1.5125172

Solid wall and open boundary conditions in hybrid recursive regularized lattice Boltzmann method for compressible flows

Physics of Fluids 31, 126103 (2019); https://doi.org/10.1063/1.5129138

\section{Soilight Highlights of the best new research in the physical sciences}

LEARN MOREI 


\title{
Topological equivalence between two classes of three-dimensional steady cavity flows: A numerical-experimental analysis ${ }^{\circledR}$
}

\author{
Cite as: Phys. Fluids 31, 123601 (2019); doi: 10.1063/1.5126497 \\ Submitted: 3 September 2019 • Accepted: 19 November 2019 • \\ Published Online: 6 December 2019
}

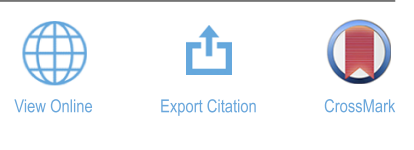

P. S. Contreras, ${ }^{1,2, a)}$ (D) I. Ataei-Dadavi, ${ }^{3}$ M. F. M. Speetjens, ${ }^{1}$ C. R. Kleijn, ${ }^{3}$ (D) M. J. Tummers, ${ }^{4}$ and H. J. H. Clercx ${ }^{2}$

\begin{abstract}
AFFILIATIONS
${ }^{1}$ Energy Technology Laboratory and J.M. Burgers Center for Fluid Dynamics, Department of Mechanical Engineering, Eindhoven University of Technology, P.O. Box 513, 5600 MB Eindhoven, The Netherlands

${ }^{2}$ Fluid Dynamics Laboratory and J.M. Burgers Center for Fluid Dynamics, Department of Applied Physics, Eindhoven University of Technology, P.O. Box 513, 5600 MB Eindhoven, The Netherlands

${ }^{3}$ Transport Phenomena Section and J.M. Burgers Center for Fluid Dynamics, Department of Chemical Engineering, Delft University of Technology, Van der Maasweg 9, 2629HZ Delft, The Netherlands

${ }^{4}$ Fluid Mechanics Section and J.M. Burgers Center for Fluid Dynamics, Department of Process and Energy, Delft University of Technology, Leeghwaterstraat 39, 2628CB Delft, The Netherlands
\end{abstract}

a) Electronic mail: p.s.contreras.osorio@tue.nl

\begin{abstract}
The present study concerns Lagrangian transport and (chaotic) advection in three-dimensional (3D) flows in cavities under steady and laminar conditions. The main goal is to investigate topological equivalences between flow classes driven by different forcing; streamline patterns and their response to nonlinear effects are examined. To this end, we consider two prototypical systems that are important in both natural and industrial applications: a buoyancy-driven flow (differentially heated configuration with two vertical isothermal walls) and a lid-driven flow governed by the Grashof (Gr) and the Reynolds (Re) numbers, respectively. Symmetries imply fundamental similarities between the streamline topologies of these flows. Moreover, nonlinearities induced by fluid inertia and buoyancy (increasing $\mathrm{Gr}$ ) in the buoyancy-driven flow vs fluid inertia (increasing Re) and single- or double-wall motion in the lid-driven flow cause similar bifurcations of the Lagrangian flow topology. These analogies imply that Lagrangian transport is governed by universal mechanisms, and differences are restricted to the manner in which these phenomena are triggered. Experimental validation of key aspects of the Lagrangian dynamics is carried out by particle image velocimetry and 3D particle-tracking velocimetry.
\end{abstract}

Published under license by AIP Publishing. https://doi.org/10.1063/1.5126497

\section{INTRODUCTION}

Transport under laminar flow conditions and chaotic advection are key to natural and industrial systems involving small-scale flows or viscous flows in, for example, microfluidics, biological flows, or geophysics. ${ }^{1,2}$ Chaotic advection is understood in the present context as purely kinematic; the solution of the advection equations (that describes the motion of passive tracers) exhibits a chaotic behavior. ${ }^{1-4}$ Closely related to chaotic advection is the "Lagrangian flow topology" (or global streamline pattern for steady flows) and its response to nonlinearities. This is fundamental to the analysis of transport phenomena.
Flows in cavities are one of the canonical configurations to study Lagrangian transport and chaotic advection in threedimensional (3D) laminar flows. ${ }^{2}$ In this context, buoyancy-driven and lid-driven flows have been considered as archetypal configurations to study fundamental aspects of advection. ${ }^{1-3,10-18}$ The main goal of this study is to investigate universal phenomena in $3 \mathrm{D}$ cavity flows, and, to this end, buoyancy-driven and lid-driven flows are adopted as representative systems. Limited insight into Lagrangian transport in this class of flows, crucial to further technological development, motivates this study.

The 3D cavity flows studied here are steady and laminar. A smooth introduction of the nonlinear effects is accomplished by 
increasing the governing parameters, which are kept well below the onset of unsteadiness so as to ensure steady flows; see, e.g., Refs. 19-21 for comparable flow conditions. The buoyancy-driven flow is generated by a horizontal temperature gradient (differentially heated cavity with two opposite isothermal vertical walls). Two driving modes are considered in the lid-driven flow: single- and doublelid forcing. This study mainly focuses on the buoyancy-driven flow in the large Prandtl number ( $\mathrm{Pr}$ ) case and its comparison with the double-lid-driven flow (antiparallel motion at the same speed of two facing walls). Former and latter flows are governed by the Grashof (Gr) and Reynolds (Re) numbers, respectively. Moreover, we consider flows in cavities of unit aspect ratio, a cubical differentially heated system, and a lid-driven flow in a square cylinder (height/diameter $=1$ ).

We further investigate the strong similarity and analogy between flows exposed in Ref. 22. This includes single-lid-driven flows (only one endwall moves) and the changes induced by doublelid-forcing. The response to nonlinearities in the buoyancy-driven flow has an equivalent counterpart in lid-driven flows: (i) The limit of vanishing $\mathrm{Gr}$ yields the same topology as the Stokes limit (Re $=0$ ) of single-lid-driven flows. (ii) Increasing $\mathrm{Gr}$ changes the flow topology in a similar way as introducing fluid inertia $(\operatorname{Re}>0)$ in the single-lid-driven case. (iii) Further increasing Gr generates a buoyancy-induced bifurcation of the streamline pattern that is reminiscent of double-lid-driven flows. The symmetry properties of the cavity flows implicate their topological similarities. This allows for a fundamental analysis while introducing nonlinear effects on two levels: (i) dynamics in the symmetry plane and (ii) 3D dynamics outside the symmetry plane.

Furthermore, a comparative numerical-experimental analysis is presented in order to validate and highlight the practical relevance of the studied Lagrangian dynamics. Particle image velocimetry (PIV) is considered to study the dynamics in the symmetry plane (in the case of the buoyancy-driven flow), and experimental analysis of tracer motion is performed by $3 \mathrm{D}$ particle-tracking velocimetry (PTV) for the double-lid-driven flow.

To date, both the buoyancy-driven and lid-driven flows have been widely considered in the literature because of their rich dynamics from a fundamental fluid mechanics point of view and their relevance to applications. However, there are still few 3D studies concerning the present cavity flows and, in particular, their Lagrangian properties. Research has shown that bifurcations occur in this type of flow, promoting complicated Lagrangian dynamics. ${ }^{19,23-26}$ However, such bifurcations in 3D have been less investigated than in the $2 \mathrm{D}$ case. This motivates the present study that examines the evolution of the streamline topologies as nonlinearities are introduced (increasing Gr, Re). Such evolution is also relevant to the onset of chaotic advection (involving break-up of transport barriers) since a substantial number of studies often consider the response of nonchaotic systems to small perturbations (e.g., Stokes flow regime).

A review of pioneering studies on the buoyancy-driven flow is presented in Refs. 22 and 27. In this configuration, extensive research has considered simplified situations in unbounded regions in which one of the aspect ratios tends to infinity. The objective in several of these studies is the understanding of the onset and the development of time-dependent flows focusing on low Pr fluids. ${ }^{27,28}$ A large body of the literature has investigated fundamental aspects in 2D and 3D rectangular cavities; see, e.g., Refs. 29-32 and references therein. Experimental visualization of flow structures can be found in Refs. 19, 33, and 34, for example. A numerical study of an airfilled cavity $(\operatorname{Pr}=0.71)$ considering bifurcations and the route to chaos with increasing values of the governing parameter is found in Refs. 25 and 26. Insight into Lagrangian transport in this configuration is relevant to buoyancy-driven flows involving additional effects, such as wall and medium radiation ${ }^{35}$ or external vibration, ${ }^{36}$ inclination of the temperature gradient, ${ }^{37,38}$ time-periodic flows composed of reoriented steady flows, ${ }^{39}$ or unsteady effects. ${ }^{40}$

Similar to the situation of the buoyancy-driven flow, 3D studies on the double-lid-driven flow remain limited. Challenges in the $3 \mathrm{D}$ case appear due to the greater topological complexity of $3 \mathrm{D}$ flows compared with their 2D counterparts. ${ }^{15,20,21,23,24}$ A recent review of studies on the 2D and 3D single- and double-lid-driven flows is presented in Ref. 18. In particular, evolution in the dynamics of the double-lid system with increasing nonlinearities in $2 \mathrm{D}$ cavities and in a 3D spatially periodic (unbounded) flow can be found in Refs. 41 and 42 , respectively. Experimental studies on this flow configuration are performed in, e.g., Refs. 43-45.

The present work substantiates the universality of key aspects of the Lagrangian dynamics. In particular, the 3D flow topology of the double-lid-driven system is characterized by similar secondary toroidal structures as found in the buoyancy-driven flow. The appearance of these structures is understood in terms of the corresponding symmetries, and the evolution of tori families is governed by generic Hamiltonian mechanisms.

This paper is organized as follows. The flow models, tracer kinematics, and numerical methods are introduced in Sec. II. In Sec. III, the details of the experimental methods are described. The comparative numerical-experimental analysis of the flow topology and Lagrangian dynamics is discussed in Sec. IV. Conclusions are drawn in Sec. $V$.

\section{PROBLEM DEFINITION}

\section{A. Buoyancy-driven flow}

We consider the steady flow in a differentially heated cubical cavity (side length $H$ ) driven by buoyancy under laminar conditions. Two opposite vertical walls are isothermal and held at different temperatures, $T_{H}$ at the left hot wall and $T_{C}$ at the right cold wall. The other four walls are thermally insulated. Furthermore, the no-slip condition for the velocity $(\boldsymbol{u}=\mathbf{0})$ is considered on all boundaries. Gravity acts in the negative $z$-direction, and the origin of the frame of reference is located in a corner of the cube [see Fig. 1(a)]. Note that left and right walls are interchanged compared with the system considered in Ref. 22 due to practical reasons in the laboratory setup.

The fluid is assumed to be Newtonian with constant physical properties except for the density in the buoyancy term following the Boussinesq approximation. The steady buoyancy-driven flow at strongly laminar conditions is governed by the mass, momentum, and energy conservation equations in the nondimensional form ${ }^{22}$

$\nabla \cdot \boldsymbol{u}=0, \quad$ Gr $\boldsymbol{u} \cdot \nabla \boldsymbol{u}=-\nabla p+\nabla^{2} \boldsymbol{u}+T \boldsymbol{e}_{z}, \quad$ Gr Pr $\boldsymbol{u} \cdot \nabla T=\nabla^{2} T$,

parameterized by the Prandtl $\mathrm{Pr}=v / \alpha$ and Grashof $\mathrm{Gr}=g \beta \Delta T H^{3} / v^{2}$ numbers. Nondimensional form (1) relies on the scaling $\boldsymbol{x}^{\prime}=H \boldsymbol{x}$, 
(a)

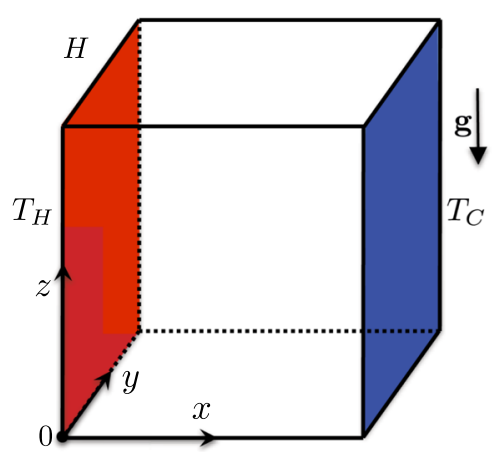

(b)

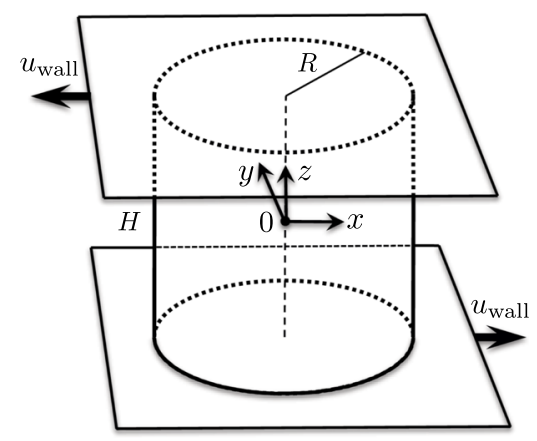

FIG. 1. Schematic of the 3D steady cavity flows: (a) Buoyancy-driven flow in a cubic cavity and (b) double-liddriven flow in a cylindrical cavity.
$\boldsymbol{u}^{\prime}=U \boldsymbol{u}, p^{\prime}=P p$, and $T^{\prime}=T_{0}+T \Delta T$. In the previous expressions, $U=g \beta \Delta T H^{2} / v, P=\mu U / H$, and $\Delta T=T_{H}-T_{C}$, with $g$ being the gravitational acceleration, $\boldsymbol{e}_{z}$ being the $z$-wise unit vector, $\mu$ being the dynamic viscosity, $v=\mu / \rho$ being the kinematic viscosity, $\beta$ being the thermal expansion coefficient, and $\alpha$ being the thermal diffusivity. Note that $\mathrm{Gr}=U H / v=$ Re; the Reynolds number and Eq. (1) govern the steady buoyancy-driven flow at low Gr. The nondimensional problem corresponds to a differentially heated cavity with unit side length and thermal boundary conditions $T=1$ at $x=0$ and $T=0$ at $x=1$.

\section{B. Double-lid-driven flow}

We consider 3D flows inside a square cylinder (radius $R$ and height $H=2 R$ ) driven by the steady and simultaneous translation of the bottom and top walls. The walls move a distance $D_{\text {wall }}$ with the same constant velocity magnitude $u_{\text {wall }}$ in the opposite direction; positive and negative $x$-directions for bottom and top walls, respectively. The mantel of the cylinder is stationary $(\boldsymbol{u}=\mathbf{0}$ at the boundary). A schematic of the configuration is shown in Fig. 1(b). The flow is governed by the steady and nondimensional continuity and momentum equations

$$
\nabla \cdot \boldsymbol{u}=0, \quad \operatorname{Re} \boldsymbol{u} \cdot \nabla \boldsymbol{u}=-\nabla p+\nabla^{2} \boldsymbol{u},
$$

with the Reynolds number $\operatorname{Re}=u_{\text {wall }} R / v$ as the control parameter. In Eq. (2), $\boldsymbol{u}$ and $p$ are the nondimensional fluid velocity and pressure, respectively. This assumes a dominance of viscous over inertial forces and the following scaling $\boldsymbol{x}^{\prime}=R \boldsymbol{x}, \boldsymbol{u}^{\prime}=u_{\text {wall }} \boldsymbol{u}, p^{\prime}=P p$ with $P=u_{\text {wall }} \rho v / R$. The cylinder $\mathcal{C}:[r, \theta, z]=[0,1] \times[0,2 \pi] \times[-1,1]$ is the associated flow domain. Note that the origin of the frame of reference is chosen to be at the center of the cylinder [Fig. 1(b)]. The nondimensional wall displacement is $D=D_{\text {wall }} / R$.

The single-lid-driven flow is governed by Eq. (2) with the boundary condition at the top wall, $\boldsymbol{u}_{\text {top }}=\mathbf{0}$. In Sec. IV A, the fundamental states of lid-driven flows are presented; the flow topology of single-lid-driven flows and the changes induced by double-lid forcing are introduced.

\section{Tracer kinematics}

The motion of passive tracers advected by the steady velocity field $\boldsymbol{u}$ is governed by the kinematic equation with corresponding solution

$$
\frac{\mathrm{d} \boldsymbol{x}}{\mathrm{d} t}=\boldsymbol{u}(\boldsymbol{x}), \quad \boldsymbol{x}(t)=\boldsymbol{\Phi}_{t}\left(\boldsymbol{x}_{0}\right)
$$

The continuous flow $\boldsymbol{\Phi}_{t}$ describes the Lagrangian tracer trajectory from the initial $\boldsymbol{x}_{0}$ to the current position $\boldsymbol{x}(t)$. The Lagrangian fluid trajectories are described by curves $\boldsymbol{x}(t)$ parameterized by $t$. These coincide with streamlines in the present context of steady flows.

A representation of the 3D flow $\boldsymbol{x}(t)=\boldsymbol{\Phi}_{t}\left(\boldsymbol{x}_{0}\right)$ by a $2 \mathrm{D}$ map $\boldsymbol{\Phi}$ is possible due to the existence of a circulatory structure in cavity flows according to

$$
\boldsymbol{x}_{k+1}=\boldsymbol{\Phi}\left(\boldsymbol{x}_{k}\right),
$$

with $\boldsymbol{x}_{k}=\left(y_{k}, z_{k}\right)$ being the $k$ th intersection of the 3D fluid trajectory starting at $\boldsymbol{x}_{0}$ with a given plane. The intersections of the streamline with this plane form a sequence of planar positions and constitute the corresponding Poincaré section. ${ }^{22}$ Both representations of the dynamics in terms of 3D streamlines and Poincaré sections will be employed in this study.

\section{Numerical methods}

The numerical simulation of the flow fields governed by Eqs. (1) and (2) is performed using the commercial CFD finite element package COMSOL Multiphysics. Standard settings are considered using the modules Laminar Flow and Heat Transfer in Fluids. In the case of the lid-driven flow, a Physics-controlled mesh with an extra fine element size is used. The computational mesh consists of $\mathcal{O}\left(1 \times 10^{6}\right)$ elements. The tolerance factor for the nonlinear solver is set to yield residuals of $\mathcal{O}\left(10^{-10}-10^{-12}\right)$. Similar solver properties are considered for the buoyancy-driven flow; see Ref. 22 for details.

Lagrangian tracer paths are determined by numerical integration of the advection equation (3) using a dedicated tracking algorithm implemented in MATLAB. ${ }^{22}$ Integration of the kinematic equation uses an explicit third-order Taylor-Galerkin scheme with adaptive step size. The reliable isolation of the fundamental topological structure by the considered numerical scheme (consistent with theoretical considerations) indicates the requirement of an adequate degree of resolution (see the discussion below).

In Ref. 22, a comparative analysis of the particle tracking scheme using the COMSOL field and its projection onto a divergence-free basis constructed from Chebyshev polynomials (spectral approach) is performed. The COMSOL field is able to 
display fundamental topological properties; the employed numerical scheme using the COMSOL field adequately resolves the formation of closed streamlines for the Stokes flow and the response to perturbations following Hamiltonian mechanisms (e.g., formation of toroidal structures). Computational departures from the incompressibility constraint in the COMSOL field lead, however, to numerical artifacts in the Lagrangian dynamics for long integration times. This involves poor resolution of the small-scale features in the Poincaré sections for further increasing the governing parameter (e.g., islandchain formation). The projected field satisfies incompressibility up to machine accuracy and enables reliable simulation of, in particular, the small-scale dynamics.

Based on the previous considerations, we present in Sec. IV a detailed numerical analysis of the buoyancy-driven and lid-driven flows. This includes the main features of the Lagrangian dynamics using the COMSOL field (resolution of the fundamental topological properties such as relevant structures and bifurcations of the streamline patterns) and long-term dynamics and small-scale features of the Poincaré sections of the buoyancy-driven flow using the spectral representation of the COMSOL field according to Ref. 22. The numerical study of flows concerns a qualitative comparison between the flow topology and associated structures. All results are critically compared with theoretical predictions based on the symmetries governing the Lagrangian dynamics (Sec. IV A) and experimental measurements when possible (see Secs. III, IV B 1, and IV C 2). Regarding the double-lid-driven flow, the particle tracking performance is examined in the Appendix. This includes a comparison between the streamline patterns obtained with the COMSOL field and a semianalytical solution $(\mathrm{Re}=0)$ and computation of the Lagrangian dynamics with increased mesh resolution $\mathcal{O}\left(3 \times 10^{5}\right)$ vs $\mathcal{O}\left(10^{6}\right)(\operatorname{Re}>0)$. This reveals adequate resolution and robustness of the simulated topological features.

\section{EXPERIMENTAL METHODS}

\section{A. Buoyancy-driven flow}

The laboratory setup consists of a cubical convection cell $(H=77 \mathrm{~mm})$. This setup is essentially a modified version of that utilized in Refs. 46 and 47 [see the sketch in Fig. 2(a)]. Copper plates serve as isothermal hot and cold walls (left and right vertical walls, respectively). The other walls are made of glass. The left wall is heated using an electrical heating foil (Minco HK5955) attached to the copper plate. The right wall is cooled using water from a thermostatic bath (Julabo FP51) flowing through channels inside the wall.

The working fluid is glycerol (Boom B.V.), with density $\rho$ $=1260 \mathrm{~kg} \mathrm{~m}^{-3}$ and $\operatorname{Pr} \sim 1.1 \times 10^{4}\left(\alpha=9.45 \times 10^{-8} \mathrm{~m}^{2} / \mathrm{s}, v=1.04\right.$ $\left.\times 10^{-3} \mathrm{~m}^{2} / \mathrm{s}\right)$. The temperature difference between the vertical walls is varied in the interval $\Delta T \in[1.3,21.5]^{\circ} \mathrm{C}$ (corresponding to $\mathrm{Gr} \in[2.6,43])$. [The analysis in Sec. IV B 1 mainly concentrates on small $\mathrm{Gr}\left(\Delta T \leq 3.3^{\circ} \mathrm{C}\right)$ in order to reduce the effects of the temperature dependence of the fluid viscosity. Nonconstant viscosity effects are mentioned as source of the differences between experiments and simulations.] The temperatures of the hot and cold plates are kept symmetrically about the laboratory ambient temperature of $22^{\circ} \mathrm{C}$ in order to minimize heat losses to the surroundings. The temperature of the vertical walls is monitored using Pt100 sensors (Omega Engineering) with an inaccuracy of $0.03^{\circ} \mathrm{C}$. The maximum observed nonuniformities of wall temperatures (examined by measuring the temperature at different locations in the plates) are $\sim 0.03^{\circ} \mathrm{C}$ and $\sim 0.01^{\circ} \mathrm{C}$ for heated and cooled plates, respectively. The Pt100 sensors are embedded in designed holes inside the copper plates; temperatures are collected by a data acquisition module and monitored using a logging software. ${ }^{46}$ (Once the measured temperatures do not vary more than $0.01{ }^{\circ} \mathrm{C}$ for a time duration of at least $15 \mathrm{~min}$, the average temperature obtained by two sensors in each copper plate is considered as the wall temperature.)

Particle image velocimetry (PIV) measurements are performed to obtain highly resolved $2 \mathrm{D}$ velocity fields in the midplane of the cavity [Fig. 2(a)]. A light sheet of approximately $2 \mathrm{~mm}$ thickness is created using a diode-pumped solid-state laser, a wavelength of $532 \mathrm{~nm}$, and a power of $0.8 \mathrm{~W}$ (Pegasus). A cylindrical lens and a mirror are used in order to illuminate the convection cell from the top.

The fluid is seeded with hollow glass spheres (density $\rho_{p}$ $=1100 \mathrm{~kg} \mathrm{~m}^{-3}$, mean diameter $d_{p}=10 \mu \mathrm{m}$, Dantec Dynamics). The gravitationally induced particle velocity is $U_{p}=\frac{\left|\rho_{p}-\rho\right|}{18 \mu} g d_{p}^{2} \sim 7$ (a)

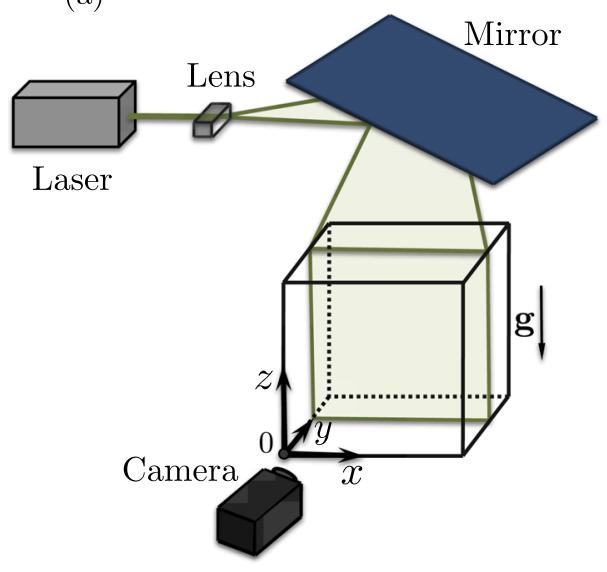

(b)

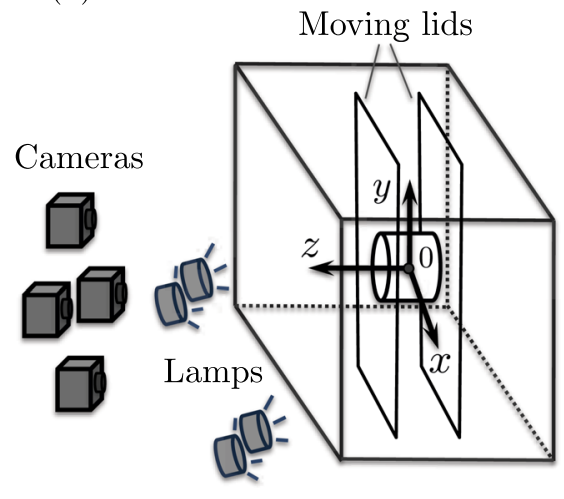

FIG. 2. Schematic of the laboratory setups: (a) PIV in the buoyancy-driven flow and (b) PTV in the double-lid-driven flow. 
$\times 10^{-9} \mathrm{~m} / \mathrm{s}$. Typical experimental velocities are $U_{\exp } \sim 50 \mu \mathrm{m} / \mathrm{s}$ and $270 \mu \mathrm{m} / \mathrm{s}$ in the high-velocity regions for minimum and maximum Gr, respectively. Therefore, $U_{p} / U_{\exp } \sim 10^{-4}$ and settling effects can be neglected. Since the particle Stokes number $S t=T_{p} / T_{f} \approx 0$, particles can be considered passive tracers, with $T_{p}=\frac{\rho_{p} d_{p}^{2}}{18 \mu}$ being the particle response time and $T_{f}=H / U_{\exp }$ being the characteristic time scale in the flow.

Particle images are recorded using a PIV camera with 1376 $\times 1040$ pixels resolution (LaVision). Velocity vectors are calculated from the raw images based on a multipass cross-correlation with final interrogation windows of $16 \times 16$ pixels and an overlap of $50 \%$ using the LaVision software (Davis 8.4.0). The time difference between consecutive images is adjusted to have a maximum particle image displacement of about 6 pixels at each experiment for a fixed temperature difference.

The mean velocity fields are determined by averaging over 3600 instantaneous velocity fields in the steady state. The uncertainty of the mean PIV velocity vectors is less than $1 \%$ of the maximum velocity in the cavity. Typical duration of experiments is between $5 \mathrm{~h}$ (at the minimum $\Delta T$ ) and $2 \mathrm{~h}$ (at the maximum $\Delta T$ ). From the calculated velocity fields, the streamlines of the steady flow are obtained using the before mentioned LaVision software with standard settings of the interpolation factor.

\section{B. Double-lid-driven flow}

Experimental analysis of tracer motion in the double-lid-driven flow is carried out by $3 \mathrm{D}$ particle-tracking velocimetry (PTV) using the laboratory setup introduced in Ref. 48 . It consists of a container filled with water $\left(v \sim 10^{-6} \mathrm{~m}^{2} / \mathrm{s}\right)$ into which a transparent Perspex cylinder $(R=35 \mathrm{~mm}, H=70 \mathrm{~mm})$ is submerged [see Fig. 2(b)]. Wall displacement is $D_{\text {wall }}=200 \mathrm{~mm}$ (this is restricted by the finitesize walls/container). The wall velocity is varied in the interval $u_{\text {wall }}$ $\epsilon[0.5,1] \mathrm{mm} / \mathrm{s}$ with an inaccuracy below $0.1 \%$. This yields an experimental Reynolds number in the interval $\operatorname{Re} \in[17.5,35]$.

The fluid is seeded with fluorescent polyethylene particles (density $\rho_{p}=1002 \mathrm{~kg} \mathrm{~m}^{-3}$, diameter $d_{p}=75-90 \mu \mathrm{m}$ ), which are illuminated by four LED arrays. Salt is added to water in order to nearly match the density of the tracer particles, resulting in $\rho=1001.7$ $\mathrm{kg} \mathrm{m}^{-3}$. The tracers are tracked by four CCD cameras, 8-bit 1600 $\times 1200$ pixels (MegaPlus II ES2020, Redlake) with B+W (orange 550) filters (Schneider Optische Werke $\mathrm{GmbH}$ ), recording at a frame rate of $f=0.5 \mathrm{~Hz}$ synchronous with the illumination. The particle tracking velocimetry algorithm developed at ETH (Switzerland) is applied to recover the $3 \mathrm{D}$ tracer trajectories. ${ }^{49,50}$ A low seeding density ( $~ 50$ particles) facilitates particle matching and isolation of complete trajectories (total duration of the experiment).

The Stokes number equals $\mathrm{St}=T_{p} / T_{f} \sim \mathcal{O}\left(10^{-5}\right)$ and thus particles can be considered passive with $T_{p}=\frac{\rho_{p} d_{p}^{2}}{18 \rho v}$ being the particle inertial response time and $T_{f}=R / u_{\text {wall }}$ being the characteristic flow time scale. The ratio particle velocity $U_{p}=\left|\rho_{p}-\rho\right| \frac{g d_{p}^{2}}{18 \rho v}$ to characteristic flow velocity amounts to $U_{p} / u_{\text {wall }} \sim \mathcal{O}\left(10^{-3}\right)$ and settling can be neglected. Moreover, since $U_{p} \sim 1.3 \mu \mathrm{m} / \mathrm{s}$ and $T_{f} \leq 70 \mathrm{~s}$, a typical deviation in a particle trajectory $\Delta x_{p} \equiv U_{p} T_{f} \sim 0.1 \mathrm{~mm}$ is obtained.

Since the Strouhal number is $\mathrm{Sr}=T_{v} / T_{\exp } \in[3,6]$, unsteady transient effects are expected in the experiments, with $T_{v}=R^{2} / v$
$=1225 \mathrm{~s}$ being the viscous time scale and $T_{\exp }=D_{\text {wall }} / u_{\text {wall }}$ $\epsilon[400,200] \mathrm{s}$ being the forcing time scale. The initial time lapses, associated with the essentially time-dependent acceleration stage, are excluded from the experimental trajectories presented in Sec. IV C 2 in order to diminish the influence of transients. For the experimental velocity range, the considered time span $\tau$ (tracking interval) varies from $70 \mathrm{~s}$ to $40 \mathrm{~s}$ for minimum and maximum $u_{\text {wall }}$, respectively. It was verified numerically (by monitoring the time evolution of the velocity components at different locations in the cavity and instantaneous flow patterns) that during the considered experimental tracking intervals, the experimental tracer trajectories should approach the final streamline patterns (essential for the purpose of this investigation). This is elaborated below.

The computations reveal that transients can be neglected after $\sim 300 \mathrm{~s}$ for the considered velocity range. For the minimum wall velocity, this means that the experimental tracer trajectories represent the streamlines in the steady state. For the higher explored wall velocities, the numerical analysis reveals that convergence to the steady streamline topology occurs relatively fast and is reached earlier than that of the velocity field itself. That is, small variations in the velocity field (caused by transients) do not produce a change in the streamline topology (differences are entirely quantitative). A qualitative comparison between simulated (steady state) and experimental tracer trajectories is presented in Sec. IV C 2.

Moreover, tracking of tracers without wall motion was performed in order to estimate the order of magnitude of experimental disturbances that act as a natural perturbation (e.g., thermal room conditions originated by the presence of electronic equipment). A circulatory-type of motion was observed consistent with a buoyancy-induced perturbation with corresponding drift velocity $v_{\mathrm{p}} \lesssim 0.1 \mathrm{~mm} / \mathrm{s}$. In Sec. IV C 2 , the effect of experimental disturbances is discussed.

\section{FLOW TOPOLOGY}

\section{A. Equivalences between flow classes}

We start this section by introducing key analogies between flows in terms of the governing equations (Sec. II) and symmetry properties. A summary from Ref. 22 of the buoyancy-driven flow is presented. Regarding lid-driven flows, the fundamental states of the flow topology of single-lid-driven flows and the changes induced by double-lid forcing are considered.

Nondimensional form (1) admits identification of two fundamental flow states. (i) The linear limit $\mathrm{Gr}=0$, characterized by closed streamlines. System (1) collapses on the Stokes limit $-\nabla p+\nabla^{2} \boldsymbol{u}+$ $T \boldsymbol{e}_{z}=\mathbf{0}$ and conductive limit $\nabla^{2} T=0$. (ii) The nonlinear regime $\mathrm{Gr}>0$, where generically nonclosed streamlines are present and the convective terms in the governing equations become relevant. Analogous states exist for lid-driven flows corresponding to cases $\mathrm{Re}=0$ and $\operatorname{Re}>0$ in Eq. (2), respectively. ${ }^{24,48,51}$

The topological similarity between the buoyancy-driven and lid-driven flows is caused by the corresponding symmetries. Furthermore, symmetries enable the introduction of equivalences between the streamline patterns of both flows (represented by regimes I-III in Fig. 3; see the discussion below). In this figure, the midplane portrays a "left-right" reflectional symmetry, and extra symmetries are illustrated by pair of points. Subscripts $b, s$, and $d$ 


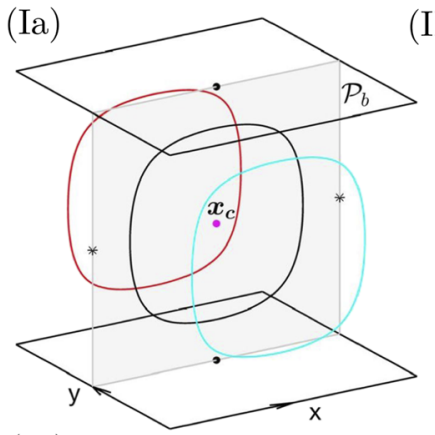

(Ib)

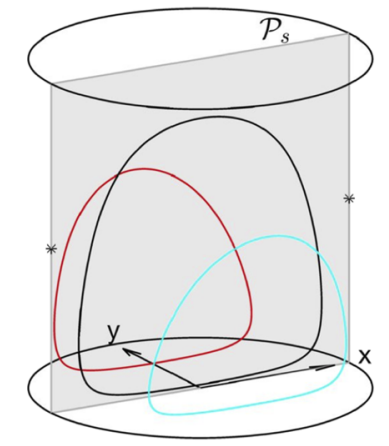

(IIa)

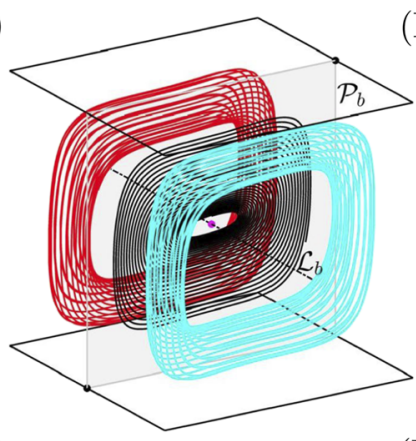

(IIb)

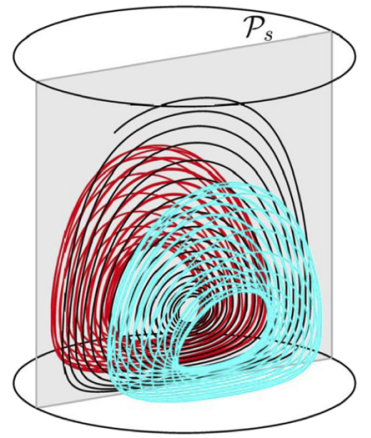

(IIIa)

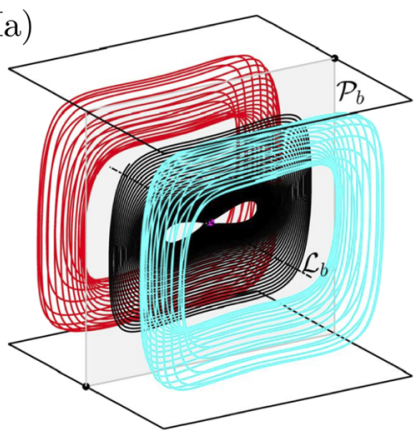

(IIIb)

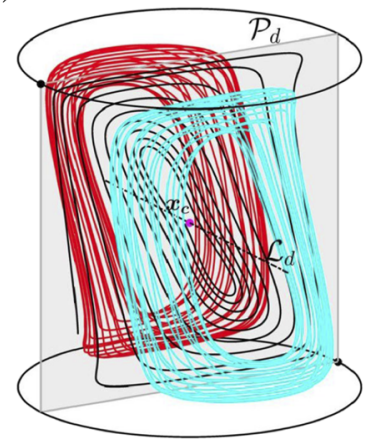

FIG. 3. Topological equivalences between flow classes. Buoyancy-driven flow (top row). Lid-driven flows (bottom row). [(lb) and (Ilb)] Single-lid-driven flow. (Illb) Double-liddriven flow. (I) $\mathrm{Gr}, \operatorname{Re}=0$. (II) $0<\mathrm{Gr}<\mathrm{Gr}^{*}$, $\operatorname{Re}>0$. (III) $\mathrm{Gr}>\mathrm{Gr}^{*}, \operatorname{Re}>0$. Symmetry plane/line $\mathcal{P}_{b, s, d} / \mathcal{L}_{b, d}$ (gray/dashed), stagnation point at the cavity center $\left(\boldsymbol{x}_{c}\right)$. Three trajectories are displayed, streamlines in $\mathcal{P}_{b, s, d}$ (black), outside $\mathcal{P}_{b, s, d}$ (cyan/red). (Illa) and (Illa) $\operatorname{Pr}=1.1 \times 10^{4}$. (IIa) $\mathrm{Gr}=2.6$. (IIla) $\mathrm{Gr}=4.6$. (Ilb) $\operatorname{Re}=10$. (IIllb) $\operatorname{Re}=100$. Pairs of points in (la), (lb) and (Ila), (Illa), and (IIlb) denote symmetries $\boldsymbol{S}_{x, z}, \boldsymbol{S}_{x}$ and $\boldsymbol{S}_{c}$, respectively.

are used to indicate the buoyancy-driven and single- and doublelid-driven flows, respectively.

The buoyancy-driven flow (1) displays the following two symmetries in the general case $\mathrm{Gr}>0 .{ }^{22,27}$ Reflectional symmetry about the midplane $y=1 / 2$ (denoted $\mathcal{P}_{b}$ hereafter) is

$$
\boldsymbol{S}_{\mathcal{P}}:(x, y, z) \rightarrow(x, 1-y, z), \quad\left(u_{x}, u_{y}, u_{z}, \widehat{T}\right) \rightarrow\left(u_{x},-u_{y}, u_{z}, \widehat{T}\right)
$$

Centrosymmetry about the central transverse line $x=z=1 / 2$ (denoted $\mathcal{L}_{b}$ hereafter) is

$$
S_{\mathcal{L}}:(x, y, z) \rightarrow(1-x, y, 1-z), \quad\left(u_{x}, u_{y}, u_{z}, \widehat{T}\right) \rightarrow\left(-u_{x}, u_{y},-u_{z},-\widehat{T}\right),
$$

with $\widehat{T}=T-1 / 2$ and $\widehat{T} \boldsymbol{e}_{z}$ being the effective buoyancy force. ${ }^{22}$ Coexistence of symmetries (5) and (6) translates into symmetry $\boldsymbol{S}_{c}=\boldsymbol{S}_{\mathcal{L}} \boldsymbol{S}_{\mathcal{P}}$ about the cavity center $\boldsymbol{x}_{c}$ that is a stagnation point, $\boldsymbol{u}\left(\boldsymbol{x}_{c}\right)=\mathbf{0}$. Centrosymmetry (6) in the limit $\mathrm{Gr}=0$ divides into the reflectional symmetries

$$
S_{x}:(x, y, z) \rightarrow(1-x, y, z), \quad\left(u_{x}, u_{y}, u_{z}, \widehat{T}\right) \rightarrow\left(u_{x},-u_{y},-u_{z},-\widehat{T}\right),
$$

$$
S_{z}:(x, y, z) \rightarrow(x, y, 1-z), \quad\left(u_{x}, u_{y}, u_{z}, \widehat{T}\right) \rightarrow\left(-u_{x},-u_{y}, u_{z}, \widehat{T}\right),
$$

about planes $x, z=1 / 2$, respectively. Symmetries (5), (7), and (8) represent the constraining mechanism underlying the formation of closed symmetric streamlines; see, e.g., Fig. 3(Ia). Single-lid-driven flows in the Stokes limit possess symmetries (5) and (7) about planes $y, x=0$, respectively [see, e.g., Fig. 3(Ib)]. The absence of symmetry (8) does not affect the topological equivalence with the buoyancydriven flow.

Breakdown of symmetries (7) and (8), and (7) for Gr, Re $>0$, respectively, dictates the formation of (generically) nonclosed streamlines. The Lagrangian flow topology consists of symmetrically arranged toroidal structures (primary tori). Streamlines in the midplane show an outward spiraling motion [Figs. 3(IIa) and 3 (IIb)], corresponding to the existence of a focus-type stagnation point.

In the buoyancy-driven flow, $\boldsymbol{x}_{c}$ bifurcates from a repelling focus for $0<\mathrm{Gr}<\mathrm{Gr}^{*}$ to a saddle for $\mathrm{Gr}>\mathrm{Gr}^{*}$, with $\mathrm{Gr}^{*}$ being the bifurcation threshold [Figs. 3(IIa) and 3(IIIa)]. This bifurcation is accompanied by a pair of repelling foci in the symmetry plane. (See Ref. 22 for details regarding the manifolds of stagnation points.) The bifurcation threshold follows a hyperbolic relation

$$
\mathrm{Gr}^{*}=a \operatorname{Pr}^{b}, \text { with }(a, b)=\left(2.53 \times 10^{4},-0.97\right)
$$

and stems from limits $\operatorname{Pr} \rightarrow 0$ (the absence of convective heat transfer, fluid inertia dominates) and $\operatorname{Pr} \rightarrow \infty$ (symmetry breaking dominated by buoyancy) in Eq. (1). ${ }^{22}$ Double-lid forcing generates 
essentially the same bifurcation for lid-driven flows in the case Re $>0$; see Figs. 3(IIb) and 3(IIIb).

Moreover, the double-lid-driven flow (2) for Re $>0$ displays symmetries (5) and (6). Reflectional symmetry about the midplane $y=0$ (denoted $\mathcal{P}_{d}$ hereafter) and centrosymmetry about the central transverse line $x=z=0$ (denoted $\mathcal{L}_{d}$ hereafter). Along $\mathcal{L}_{b, d}$, the only (in general) nonvanishing velocity component is $u_{y}$ (i.e., $u_{x}=u_{z}=0$ ) due to symmetry (6).

In summary, the response to nonlinearities in the buoyancydriven flow has an equivalent counterpart in lid-driven flows (this includes single- and double-lid forcing). Equivalent flow topologies (see Fig. 3) result from the corresponding symmetries.

Two main cases can be distinguished in the buoyancy-driven flow at small and large Pr, respectively. (In Ref. 22, the considered parameter range is $10^{-2} \leq \operatorname{Pr} \leq 10^{2}$ and "large $\operatorname{Pr}$ " means $\operatorname{Pr} \geq 7$.) Only for large $\operatorname{Pr}$, the existence of secondary tori around $\mathcal{L}_{b}$ (displaying a reversed circulation) is observed. Increasing Gr causes the progressive disintegration of tori into chaotic streamlines following universal Hamiltonian mechanisms [governed by the KolmogorovArnold-Moser (KAM) and Poincaré-Birkhoff theorems]. ${ }^{4}$

The emergence of secondary tori is caused by the existence of stagnation points on $\mathcal{L}_{b}$ (whenever $u_{y}$ vanishes); details on this mechanism can be found in Ref. 22. The presence of such points results in the formation of a "separatrix" between tori families due to intricate heteroclinic manifold interactions. Moreover, the existence of stagnation points (generally) precludes the possibility of a global Hamiltonian structure in the flow domain. ${ }^{2}$ This restricts the Hamiltonian scenario to subregions (as in the case of the two tori families).

In this study, we further investigate the equivalences between flows by both numerical and experimental analyses. The study of the buoyancy-driven flow in the large Pr case is shown in Sec. IV B, followed by the dynamics of the double-lid-driven flow and its comparison with the buoyancy-driven flow in Sec. IV C. In particular, two key elements are considered: (i) the dynamics and bifurcations in the symmetry plane and (ii) the $3 \mathrm{D}$ emergence of secondary tori.

\section{B. Buoyancy-driven flow}

\section{Dynamics in the symmetry plane}

We focus hereafter on the analysis of the buoyancy-driven flow in the large $\operatorname{Pr}$ case $\left(\mathrm{Pr}=1.1 \times 10^{4}\right)$. Differences between large and small $\mathrm{Pr}$ are emphasized. In particular, the PIV experiments introduced in Sec. III A were designed to test and extend the study of the Lagrangian dynamics in the symmetry plane (Sec. IV A).

Figure 4 shows the comparison between the streamlines in the symmetry plane $\mathcal{P}_{b}$ for two values of $\mathrm{Gr}$ obtained experimentally (left panels) and numerically (right panels). The experiments clearly show the bifurcation of the central stagnation point from a focus for small Gr [Fig. 4(a)] to a saddle for larger Gr [Fig. 4(b)] accompanied by a pair of foci. In the experimental case, a collection of streamlines obtained by PIV is displayed. In the numerical simulations, representative trajectories (tracked both forward and backward in time) are shown. Different colors are used for clarity of the streamline patterns. Red and black streamlines in the right panel of Fig. 4(b) have symmetric initial positions. The numerical streamlines indicate stability of foci as discussed in Sec. IV A.

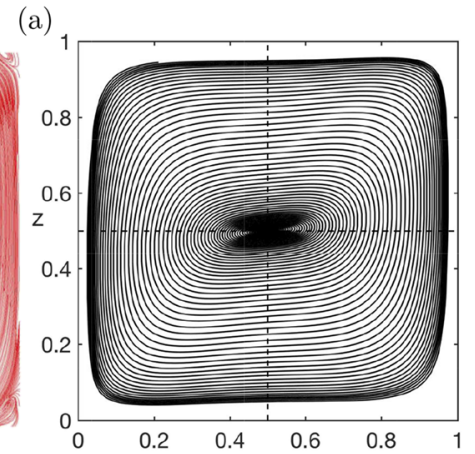

(b)
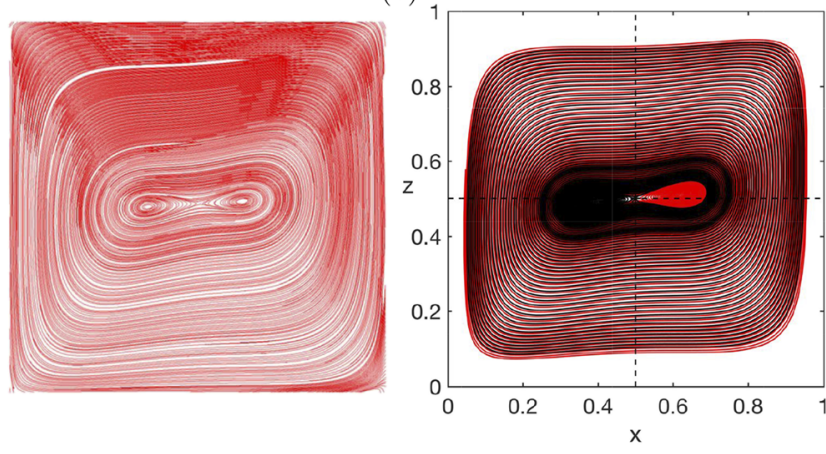

FIG. 4. Buoyancy-driven flow, $\mathrm{Pr}=1.1 \times 10^{4}$. Lagrangian fluid trajectories in the symmetry plane $(y=1 / 2)$. Experiments, streamline collection (left). Simulations, representative streamlines (colors represent different initial positions) (right). (a) $\mathrm{Gr}=2.6$. (b) $\mathrm{Gr}=4.6$

Note the typical behavior of large Pr, circulation is weak (compared with small $\mathrm{Pr}$ ) and streamlines, in consequence, display a dense winding. ${ }^{22}$ There is a good agreement between experiments and simulations, and the experimental measurements qualitatively follow the predicted Lagrangian dynamics with increasing nonlinearities.

Figure 5 shows the evolution of the Lagrangian dynamics in $\mathcal{P}_{b}$ by further increasing Gr. The experimental streamlines continue to reflect the simulated dynamics; the evolution from Figs. 4(b) to 5(a) shows an increased area associated with the pair of foci. Moreover, a good agreement is also found regarding the occurrence of a new bifurcation of the central stagnation point by further increasing Gr; see Fig. 5(b). This new bifurcation is entirely consistent with the evolution of the Lagrangian dynamics in the symmetry plane for small $\mathrm{Pr}$, and the central stagnation point changes from a saddle to a focus accompanied by two focus-saddle pairs. ${ }^{26}$ This can be clearly seen in the experiments of Fig. 5(b).

A new feature for the large Pr case can be deduced from the simulations shown in Fig. 5, the existence of an unstable limit cycle (repelling both in its interior and exterior regions, this was determined by forward and backward tracking of trajectories). The limit cycle divides the flow domain and encloses the arrangement of stagnation points [e.g., separation of red/black and blue streamlines in Fig. 5(a)]. Accompanying the limit cycle, stability reversal is observed (in comparison with lower Gr), e.g., foci in Fig. 5 
(a)
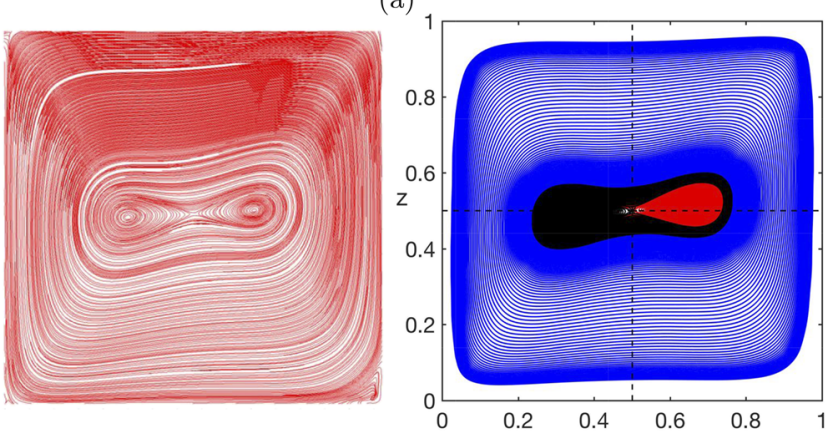

(b)
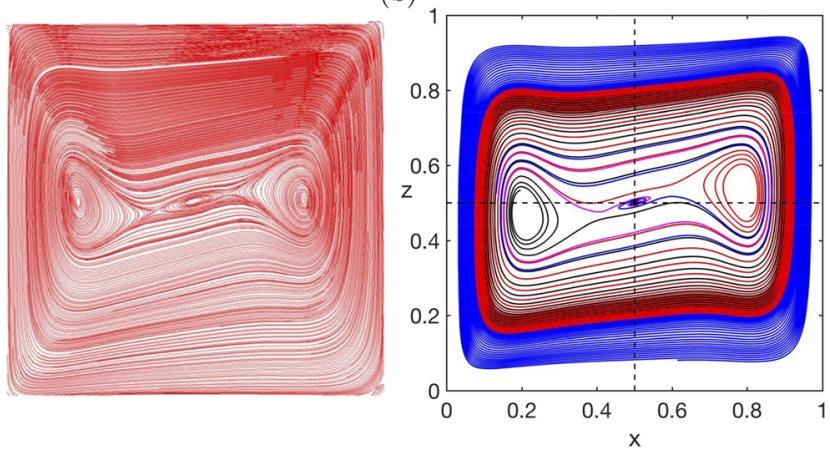

FIG. 5. Buoyancy-driven flow, $\mathrm{Pr}=1.1 \times 10^{4}$. Lagrangian fluid trajectories in the symmetry plane $(y=1 / 2)$. Experiments, streamline collection (left). Simulations, representative streamlines (colors represent different initial positions) (right). (a) $\mathrm{Gr}=6.7$. (b) $\mathrm{Gr}=43$.

(right) become attracting within the symmetry plane (determined by forward and backward tracking). With increasing $\mathrm{Gr}$, the interior region of the limit cycle increases gradually in size. The limit cycle is also found in the representative case of study with $\operatorname{Pr}=7$ in Ref. 22 and for $\operatorname{Pr}=50$ in Ref. 52 .

Symmetry $\boldsymbol{S}_{\mathcal{P}}$ restricts the dynamics in $\mathcal{P}_{b}$ to a bounded $2 \mathrm{D}$ manifold under steady conditions. The Poincaré-Bendixson theorem essentially dictates the dynamics in the symmetry plane: streamlines must describe a closed path (limit cycle) or approach a stagnation point or a limit cycle. ${ }^{53}$ Note, in particular, that chaotic streamlines are excluded within $\mathcal{P}_{b}$. An important remark is that the observed dynamics represents an entirely 3D behavior associated with the three-dimensionality of the flow domain. Streamlines remain as closed curves with increasing nonlinearities in 2D cavities; see, e.g., Refs. 29, 30, and 41. A state of the 3D flow topology characterized by closed streamlines is only present for the Stokes limit $(\mathrm{Gr}=0)$; see, e.g., Fig. 3(Ia).

The same evolution shown in Figs. 4 and 5 in terms of the stagnation points was numerically obtained in this investigation for $\mathrm{Pr}$ $=1$ and increasing $\mathrm{Gr}\left(4.9 \times 10^{3}, 4 \times 10^{4}, 3 \times 10^{5}\right)$ (as a representative example of small Pr). However, no limit cycle was found up to $\mathrm{Gr} \sim 5 \times 10^{5}$. This agrees with the analysis in the symmetry plane for $\operatorname{Pr}=0.71$ presented in Refs. 25 and 26. The existence (or absence) of the limit cycle represents a clear difference in the dynamics between large and small Pr fluids, respectively. The considered

dynamics and discussion of a Rayleigh-Bénard flow in a bounded domain in Refs. 54 and 55 suggest that a similar behavior is found in the symmetry plane of that system: existence of the limit cycle only for large Pr. Reconciliation of this phenomenon from a unified point of view of general buoyancy-driven flows in cavities is needed; however, this is beyond the present scope.

Closer examination of the experimental results displayed in Figs. 4 and 5 indicates a slight asymmetry in comparison with the theoretical and numerical results. This was further confirmed by inspection of the corresponding PIV velocity fields. Moreover, exploration of single streamlines showed no evidence of a limit cycle in the experimental data (Fig. 5, left panels).

The agreement between simulated and experimental streamline patterns indirectly suggests that departure from symmetries is weak. However, as considered in Ref. 19, temperature differences produce (local) viscosity changes of glycerol from $40 \%$ for $\Delta T=4{ }^{\circ} \mathrm{C}$ and more than $100 \%$ for $\Delta T=15^{\circ} \mathrm{C}$. This implicates a violation of the Boussinesq approximation which is expected to be the reason behind the asymmetries in the experimental streamline patterns. See also the discussion in Ref. 56 regarding non-Boussinesq effects in the present configuration. Furthermore, typical experimental disturbances such as nonideal boundary conditions are also known to have an effect that translates into discrepancies between experiments and simulations. ${ }^{19,34,57}$ This is an important issue for future research, particularly regarding $3 \mathrm{D}$ flow structures.

Agreement between experiments and simulations is shown in Fig. 6. This figure compares the bifurcation threshold $\mathrm{Gr}^{*}$ according to Eq. (9) (line) with the experimental results shown in Fig. 4 and the experimental and numerical results for $\operatorname{Pr} \sim \mathcal{O}\left(6 \times 10^{3}\right)$ of Refs. 19 and 58, respectively. (Data from Fig. 5 are also shown for completeness.) Figure 6 experimentally validates the bifurcation threshold for larger Pr. As predicted by the hyperbolic relation (9), we observe a decreasing threshold and earlier bifurcation with growing $\mathrm{Pr}$ (Sec. IV A).

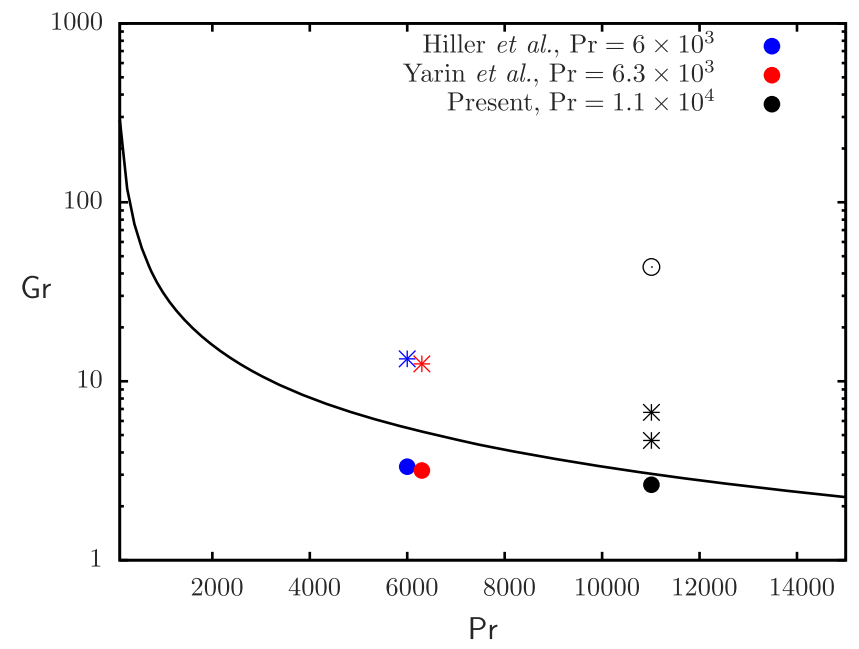

FIG. 6. Comparison between the predicted bifurcation threshold $\mathrm{Gr}^{*}$ [Eq. (9), line] and experimental and simulated data for larger Pr. Prebifurcated and bifurcated streamline patterns are indicated using solid circles and asterisks, respectively. The open circle represents the data of Fig. 5 (b). 


\section{Secondary tori}

Figure 7 presents numerical 3D tracer trajectories with increasing Gr. The primary family of tori centered on $\mathcal{L}_{b}$ (dashed line) is shown in Fig. 7(a). The emergence of a secondary family of KAM tori close to the cavity wall $(y=0)$ with increasing $\mathrm{Gr}$ is shown in the central region of Fig. 7(b). Only tracers in the region $y<1 / 2$ are shown which is sufficient due to symmetry $\boldsymbol{S}_{\mathcal{P}}$.

Figure 8 shows the Poincaré sections associated with a collection of tracer trajectories with increasing $\mathrm{Gr}$. The $z=1 / 2$ dashed line represents $\mathcal{L}_{b}$. The typical structure of the primary family of tori and the coexistence of the two tori families are shown in Figs. 8(a) and 8(b), respectively. The evolution shown in Figs. 7 and 8 confirms the generality of the existence of the secondary family of KAM tori for larger $\mathrm{Pr}$ and the response to nonlinearities of the tori families according to the dynamics presented in Sec. IV A.

Moreover, the intimate relation between the appearance of secondary tori and the existence of stagnation points on $\mathcal{L}_{b}$ (whenever $u_{y}$ vanishes; see Sec. IV A) for $\operatorname{Pr}=1.1 \times 10^{4}$ was confirmed in this study. Considering only the left half of the cavity due to symmetry $\boldsymbol{S}_{\mathcal{P}}$, velocity component $u_{y}$ continuously changes from positive to negative in the interior region (between $\mathcal{P}_{b}$ and the cavity wall) with increasing $\mathrm{Gr}$. At intermediate $\mathrm{Gr}$, stagnation points exist in the interior region corresponding to zeros of $u_{y}$. Examination of the dynamics for $\operatorname{Pr}=7$ (representative example in Ref. 22) and further increasing nonlinearities, up to $\mathrm{Gr}=1.3 \times 10^{5}$, indicates a similar evolution. This includes the dynamics in $\mathcal{P}_{b}$ (Sec. IV B 1), the Poincare sections and the evolution of the stagnation points on $\mathcal{L}_{b}$. Therefore, the described Lagrangian dynamics are expected to be generic for the large Pr case.

Furthermore, exploration of the dynamics for $\operatorname{Pr}=1$ and increasing nonlinearities (up to $\mathrm{Gr} \sim 5 \times 10^{5}$ ) confirmed that no secondary tori exist (and closely related to that, no stagnation points in the interior region on $\mathcal{L}_{b}$ appear); in this case, $u_{y}>0$ in the interior region along $\mathcal{L}_{b}$.

There are similarities between the evolution displayed by the two tori families and steady vortex cylindrical cavity flows generated by rotating one or both end covers; see Refs. 7, 59, and 60 and references therein. One or more recirculating zones appear (occupying different regions of the flow domain) depending on the governing parameters. In particular, a bifurcation described by the creation of a closed bubble-shaped region of fluid "vortex-breakdown" has been widely studied. The creation of bubbles can be analyzed by monitoring the existence of stagnation points on the cylinder axis (zeros of the axial velocity). The cylinder axis plays the role of $\mathcal{L}_{b}$ in the buoyancy-driven flow (see also Sec. IV C 3).

Analogous behavior has also been observed in flows between rotating spheres. ${ }^{59,60}$ The dynamics of the steady flow between concentric rotating spheres, or the spherical Couette flow, discussed in, e.g., Refs. 61-63, suggests that the appearance of secondary tori might be induced by changing geometrical parameters (in a wide range of Re). The flow topology can change from a "one-cell" to a "two-cell" flow pattern depending on the rotation parameters. Future efforts are needed to understand these similarities from a unified framework.

\section{Double-lid-driven flow}

In this section, the analysis of the flow topology of the doublelid-driven flow is presented. Symmetry properties (Sec. IV A) allow for a similar fundamental analysis with increasing nonlinearities as in the buoyancy-driven case. In the following, we consider first the dynamics in $\mathcal{P}_{d}$ and subsequently the essentially $3 \mathrm{D}$ dynamics outside this plane. Similarities and differences between the double-liddriven and buoyancy-driven flows are emphasized.

\section{Dynamics in the symmetry plane}

Figure 9 shows the evolution of representative numerical streamlines in the symmetry plane $\mathcal{P}_{d}$ with increasing Re. Tracers are tracked both forward and backward in time in order to determine the stability of the relevant structures (see the discussion below). Different colors (representing trajectories with different initial positions) are used for clarity of the streamline patterns.

The Stokes limit $(\mathrm{Re}=0)$ is characterized by closed streamlines [Fig. 9(a)]. In this case, the stagnation point at the cavity center is a hyperbolic saddle and there are two symmetrically arranged centers (or degenerate foci) giving rise to the "two-eddy" streamline pattern or "cat-eyes" flow structure. ${ }^{17,24,42}$ In this case, the streamline pattern is also symmetric about the planes $x, z=0$ according to symmetries (7) and (8). Combination of these extra symmetries with $\boldsymbol{S}_{\mathcal{P}}$ results in the formation of three stagnation lines (corresponding to the three stagnation points in the symmetry plane) extending along $y$. Similarly to the buoyancy-driven flow, the Stokes limit is the only state analogous to the $2 \mathrm{D}$ flow. (a)

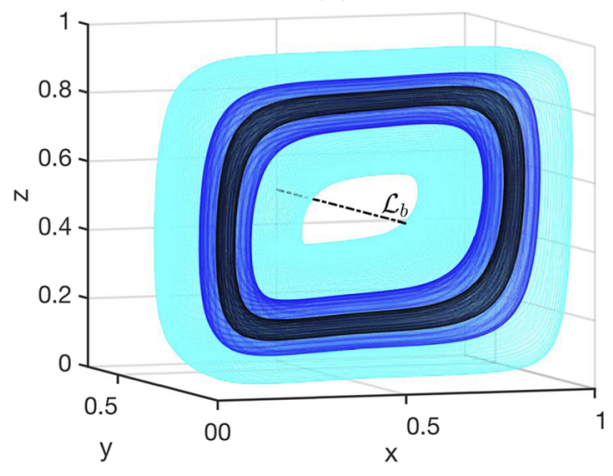

(b)

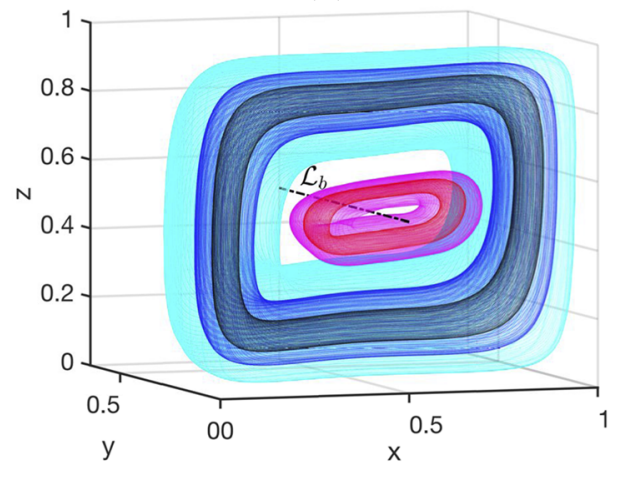

FIG. 7. Buoyancy-driven flow, $\mathrm{Pr}=1.1$ $\times 10^{4}$. Emergence of a secondary family of KAM tori with increasing $\mathrm{Gr}, \mathcal{L}_{b}$ (dashed line). Representative tracer trajectories (distinguished by color for clarity of the concentric structure) with different initial positions. (a) $\mathrm{Gr}=2.6$. (b) $\mathrm{Gr}$ $=4.6$. 
(a)

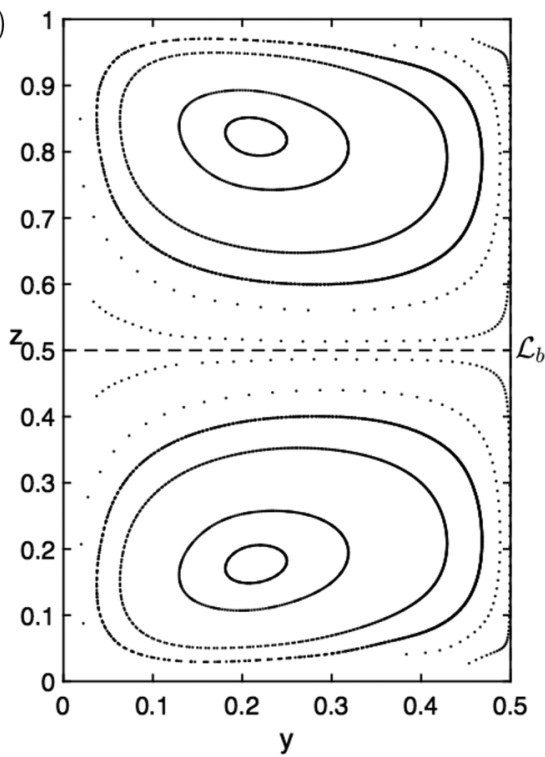

(c)

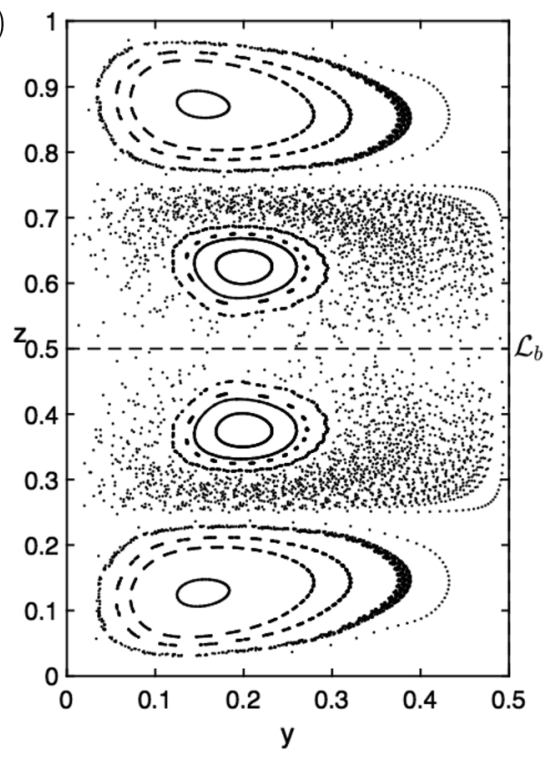

(b)

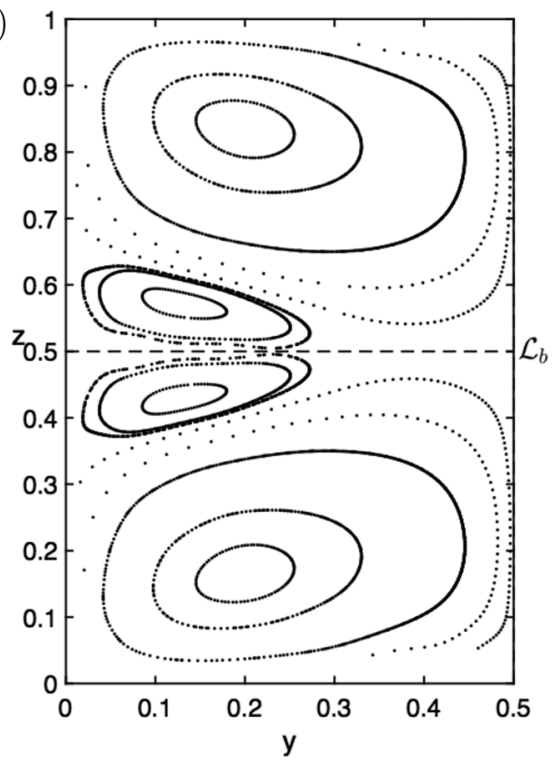

(d)

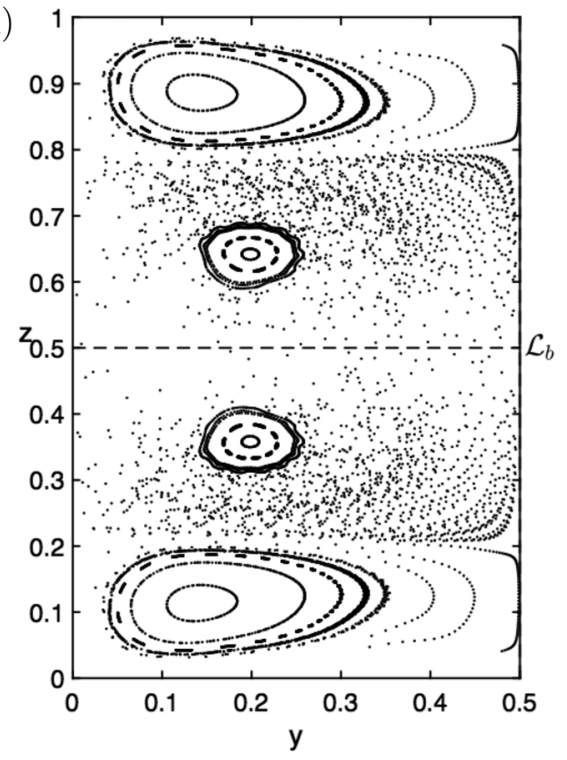

FIG. 8. Buoyancy-driven flow, $\mathrm{Pr}=1.1$ $\times 10^{4}$. Evolution of simulated toroidal structures with increasing $\mathrm{Gr}, \quad \mathcal{L}_{b}$ (dashed line). Visualization by intersections with the plane $x=1 / 2$ (Poincaré section). (a) $\mathrm{Gr}=2.6$. (b) $\mathrm{Gr}=4.6$. (c) $\mathrm{Gr}=20$. (d) $\mathrm{Gr}=43$.
Increasing Re eliminates the two extra symmetries of the Stokes limit in favor of $\boldsymbol{S}_{\mathcal{L}}$ and consequently the constraining mechanism underlying the formation of closed streamlines vanishes. The twoeddy structure is perturbed, and the dynamics is characterized by the appearance of a pair of repelling foci with a saddle at the cavity center. Typical streamlines in this case are shown in Figs. 9(b) and $9(\mathrm{c})$. [We show representative streamlines to highlight the Lagrangian dynamics; red and black streamlines in Figs. 9(b)-9(d) have symmetric initial positions.] This is entirely equivalent to the dynamics shown in Fig. 4(b) of the saddle-foci arrangement in the buoyancy-driven flow [Figs. 3(IIIa) and 3(IIIb)].

In the double-lid-driven flow, increasing Re reduces the area of influence of the pair of foci. Eventually, an unstable limit cycle appears surrounding the arrangement of stagnation points plus stability reversal of the foci that become attracting [Fig. 9(d)]. This behavior is completely analogous to the dynamics shown in Fig. 5(a) in the buoyancy-driven case.

Further increasing Re causes a bifurcation of the central stagnation point that becomes an attracting focus [Fig. 9(e)], and the unstable limit cycle remains. Figure 9 (f) shows the evolution with further increasing nonlinearity; the central focus becomes repelling, and two limit cycles exist: a stable one (inner, attracting in both interior and exterior regions) and an unstable one (outer, repelling in both interior and exterior regions). First exploration suggests that this behavior remains up to $\mathrm{Re}=1000$. The evolution shown in Figs. $9(\mathrm{e})$ and $9(\mathrm{f})$ reveals a bifurcation resulting in a streamline 

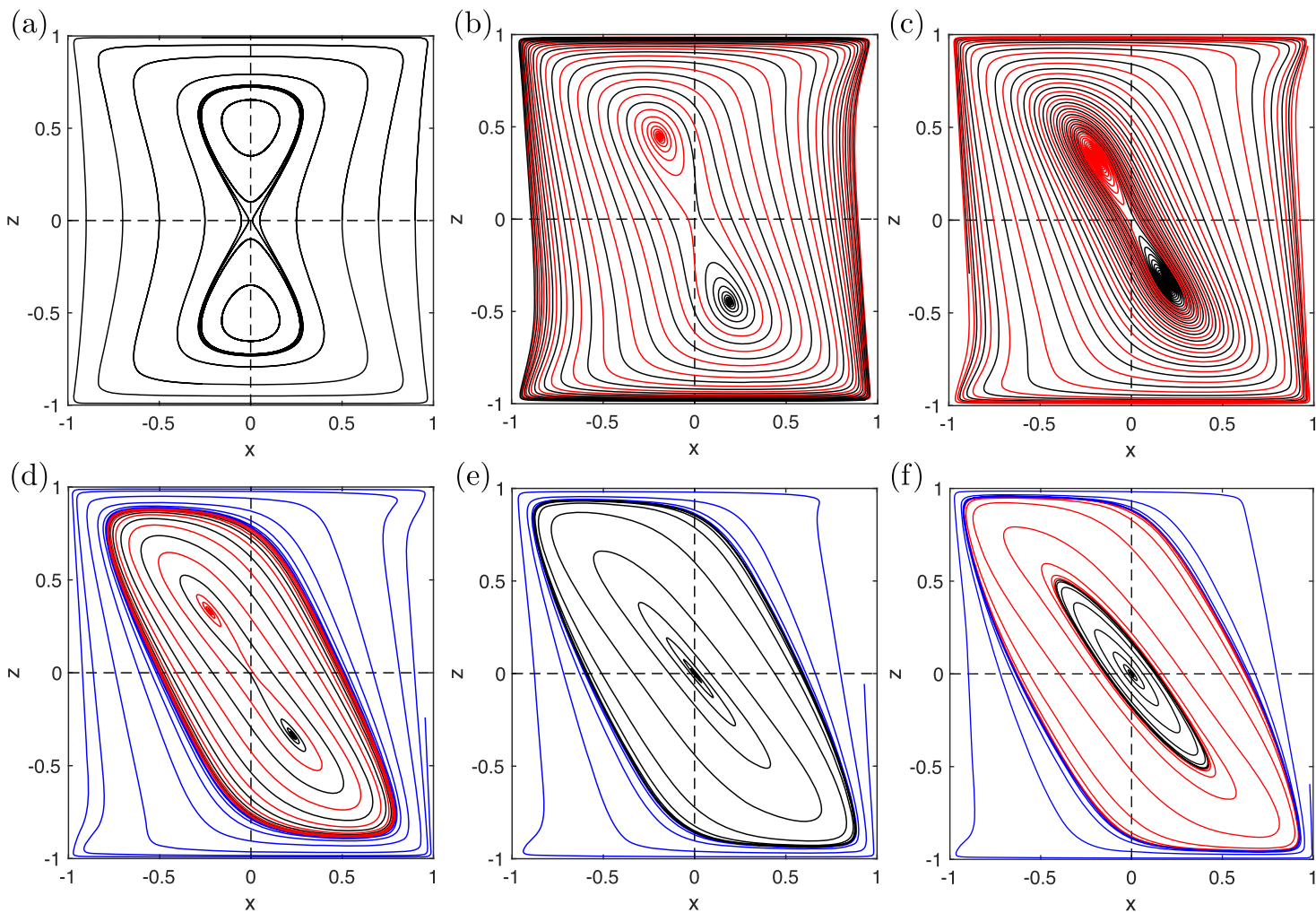

(f)

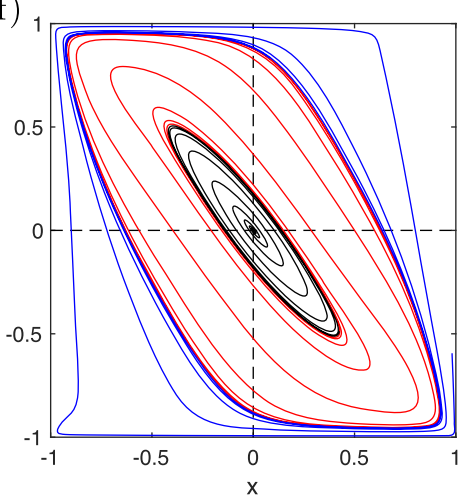

FIG. 9. Double-lid-driven flow with increasing Re. Representative simulated streamlines with different initial positions (distinguished by color for clarity) in the symmetry plane $(y=0)$. (a) $\operatorname{Re}=0$. (b) $\operatorname{Re}=50$. (c) $\operatorname{Re}=100$. (d) $\operatorname{Re}=200$. (e) $\operatorname{Re}=350$. (f) $\operatorname{Re}=500$.

pattern consisting of multiple limit cycles. (See Refs. 5 and 64 for a discussion regarding similar bifurcations.) In particular, the transition in the central part of Figs. 9(e) and 9(f) from an attracting focus at the cavity center to a repelling focus accompanied by the stable inner limit cycle with increasing Re shows similarities with a supercritical Hopf bifurcation. ${ }^{4}$ This should be explored in more detail in future studies.

Completely equivalent to the dynamics of the buoyancydriven flow, the Poincaré-Bendixson theorem governs the dynamics in the symmetry plane. However, a one-to-one correspondence between both flows with increasing nonlinear effects restricts to the foci-saddle structure with and without the unstable limit cycle [Figs. 4(b), 5(a), and 9(b)-9(d)]. Further increasing nonlinear effects displays clear differences between flows in the symmetry plane: on the one hand, the double-lid-driven flow is characterized by a single focus stagnation point at the cavity center and multiple limit cycles [Fig. 9(f)]. On the other hand, multiple bifurcations of the central stagnation point occur accompanied by focussaddle pairs for the buoyancy driven flow [see, e.g., Fig. 5(b) and Refs. 25 and 26].

However, the 3D dynamics outside the symmetry plane of the double-lid-driven flow (Sec. IV C 3) shows an analogous behavior to the one presented in Sec. IV B 2 for the buoyancy-driven flow and increasing nonlinearities (particularly regarding the existence of secondary tori).

\section{Experimental 3D flow structure}

In this section, a qualitative analysis of $3 \mathrm{D}$ tracer motion and governing symmetries is presented for $\operatorname{Re}>0$. Figure 10 shows typical 3D experimental tracer trajectories obtained by PTV and their simulated counterparts. An overlay of trajectories is exposed, revealing a general agreement between both cases. The streamline patterns shown in Fig. 10 begin to delineate the typical perturbed two-eddy structure. In particular, Figs. 10(a) and 10(c) give a first clear indication of the effect of increasing Re, an inclination of the two circulatory regions.

Natural disturbances in the experiments can induce departures from the predicted dynamics. Experimental imperfections act as natural perturbations of the (idealized) unperturbed state (Sec. III B). Even a minute asymmetry produced by the nonparallel translation of the moving lids, for example, can trigger symmetry breaking (this was observed numerically in this investigation). These experimental disturbances are expected to be the reason behind the deviations from the simulated tracer trajectories shown in Fig. 10.

Figure 11(a) shows the experimental trajectories close to the midplane $\mathcal{P}_{d}$ in the region $|y| \leq 0.25$. Streamlines exhibiting midplane crossing (i.e., symmetry breaking) are highlighted in blue (thick markers). However, displacement along $y$ is considerably less than in the other directions. The crossing angle $\delta$ for the two tracers moving vertically near the mantle of the cylinder is $\delta \simeq 26^{\circ}$ and 
(a)

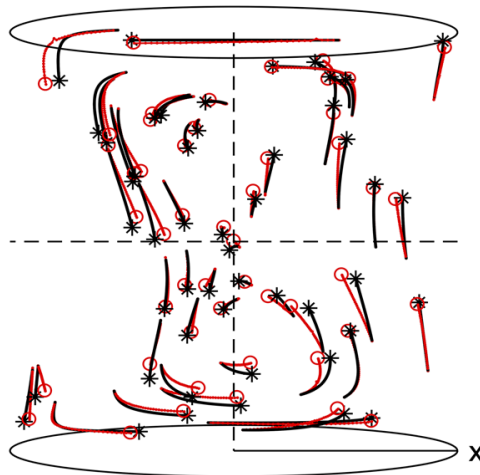

(b)

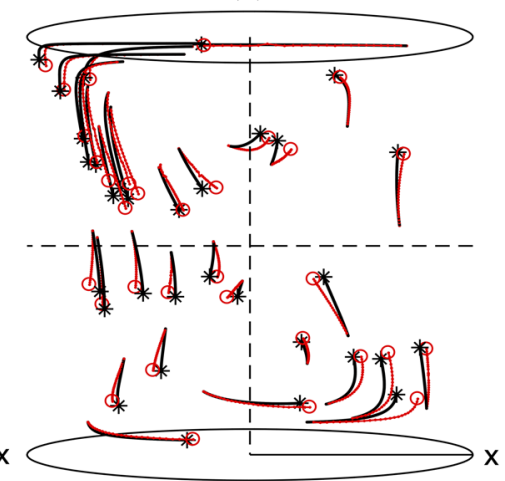

(c)

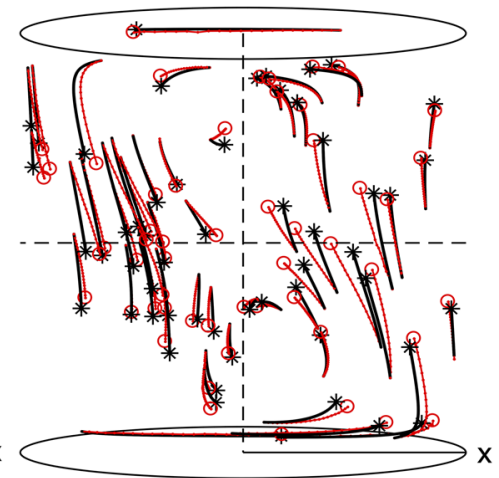

FIG. 10. Double-lid-driven flow. Comparison between 3D experimental (red) and numerical (black) tracer trajectories. $(\mathrm{a}) \mathrm{Re}=17.5 .(\mathrm{b}) \mathrm{Re}=28$. (c) Re $=35$. Open circles (red) and asterisks (black) indicate the corresponding final tracer positions.

$\delta \lesssim 7^{\circ}$ for the others. This reflects the fact that even if no exact symmetry exists in the experimental streamlines, perpendicular motion is limited and tracers remain near the midplane.

Figure 11(b) presents a side view of measured streamlines and their reflected counterparts (according to $\mathcal{S}_{\mathcal{L}}$ ) in the region $|y| \leq 0.45$ for clarity. The two circulatory regions characterizing the foci-saddle structure are delineated and a global consistency in tracer motion is visible. A similar behavior is found for the other explored values of Re. The previous discussion strongly suggests that experimental departure from symmetries is weak.

\section{Secondary tori}

The evolution of typical 3D streamlines (and corresponding Poincaré sections, $z=0$ ) obtained by numerical simulations with increasing $\mathrm{Re}$ is shown in Fig. 12. Only tracers on one half of the cavity $(y>0)$ are shown which is sufficient due to the symmetry $\boldsymbol{S}_{\mathcal{P}}$ (streamlines are colored differently for clarity). For low Re, a single family of toroidal structures exists (primary tori) [Figs. 12(a) and 12(d)]. The emergence of a secondary family of tori around $\mathcal{L}_{d}$ (represented by the $x=0$ dashed line) with increasing nonlinearity can be seen in the center and right panels of Fig. 12. The region occupied by the secondary tori closely matches the position(s) of the limit cycle(s) that exist in the symmetry plane (Fig. 9). First inspection suggests that the persistence of the two families of KAM tori remains up to $\operatorname{Re}=1000$.

The dynamics of the Poincaré sections in Fig. 12 is fully consistent with the Hamiltonian scenario of the buoyancy-driven flow (Sec. IV A). Increasing Re progressively triggers torus breakdown accompanied by growing chaotic regions. Moreover, entirely equivalent to the behavior found in the buoyancy-driven case (Sec. IV A), a circulation in a reversed direction corresponding to the families of tori is observed: counterclockwise and clockwise directions for primary and secondary tori in the region $x>0$ in Fig. 12, respectively. Because of the similarities between flows (Figs. 12, 7, and 8), a similar behavior regarding the separatrix between tori families (Sec. IV A) is also suspected to occur here (conclusive establishment is outstanding).

As in the buoyancy-driven flow, exploration of $u_{y}$ enables us to detect stagnation points on $\mathcal{L}_{d}$. A similar evolution is found for the (a)

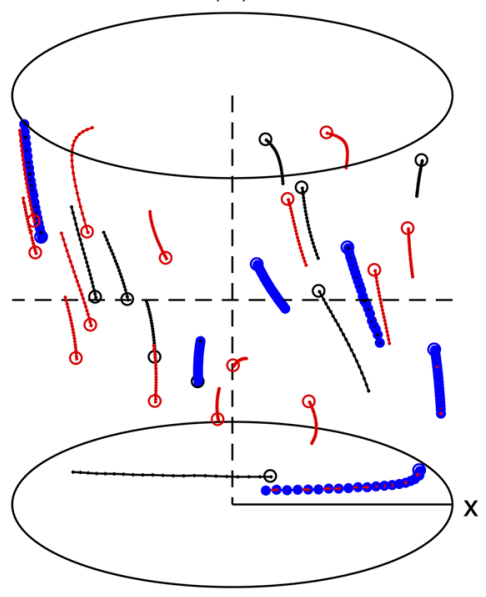

(b)

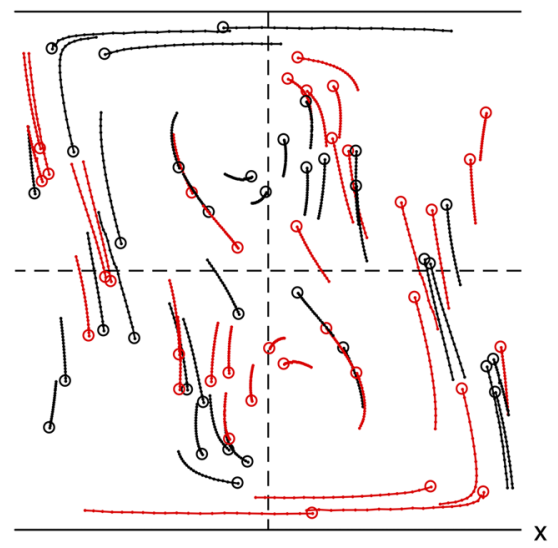

FIG. 11. Double-lid-driven flow, $R e=35$. (a) Trajectories near $\mathcal{P}_{d},|y| \leq 0.25$. Black $(y>0)$, red $(y<0)$, and blue/thick (midplane crossing). (b) Side view, $|y|$ $\leq 0.45$. Measured trajectories (red) and reflected trajectories according to $\mathcal{S}_{\mathcal{C}}$ (black). Circles indicate the final tracer positions. 
(a)

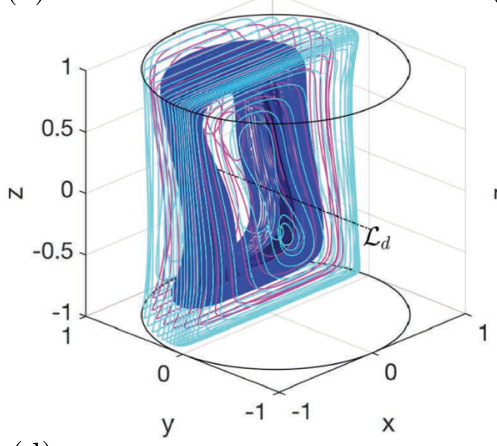

(d)

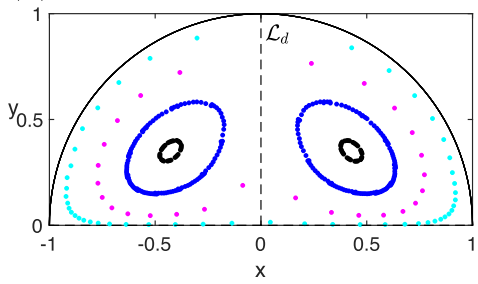

(b)

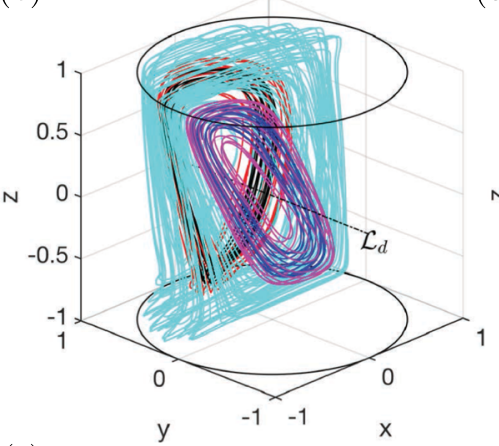

(e)

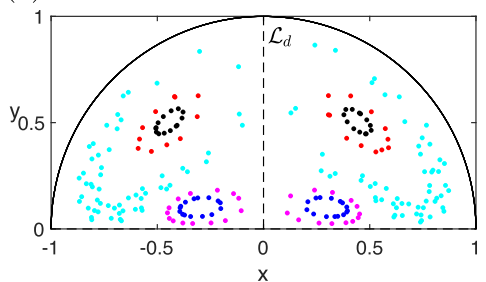

(c)

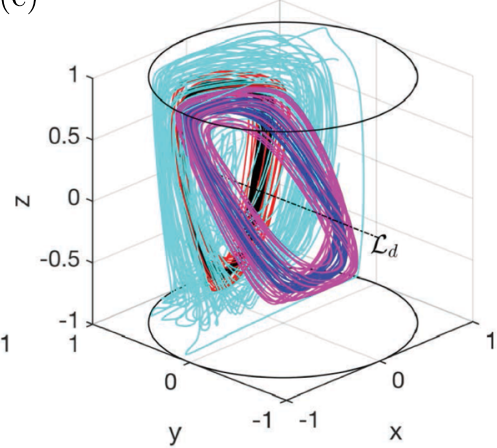

(f)

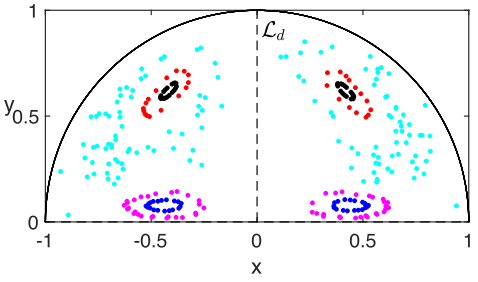

FIG. 12. Double-lid-driven flow. Emergence of a secondary family of KAM tori with increasing $\mathrm{Re}, \mathcal{L}_{d}$ (dashed line). Representative tracer trajectories (distinguished by color for clarity of the concentric structure) with different initial positions (top) and corresponding Poincaré sections, $z=0$ (bottom). [(a) and (d)] $\operatorname{Re}=50$. [(b) and (e)] $\operatorname{Re}=200$. $[(c)$ and $(f)] \operatorname{Re}=500$.

double-lid-driven flow with increasing nonlinearities. However, the position of the stagnation point on $\mathcal{L}_{d}$ at, e.g., $\mathrm{Re}=200$ (close to $\mathcal{P}_{d}$ ) implies a difference between the double-lid-driven and buoyancydriven flows. Secondary tori in this case do not appear near the bounding cavity wall and gradually expand toward $\mathcal{P}_{d}$ with increasing nonlinearity; they appear and remain close to the symmetry plane; see Fig. 12.

Nevertheless, the dynamics shown in Fig. 12, and the closely related evolution of stagnation points on the symmetry line, reinforces the generality of the existence of the secondary family of tori with increasing nonlinearities in flows in bounded domains.

Moreover, the evolution displayed in Fig. 12 (bottom) is similar to the evolution presented in Ref. 59 of steady vortex breakdown flows in a cylindrical cavity with corotating top and bottom walls at the same angular velocity and increasing Re. Secondary tori behave as vortex bubbles, and the axis of the rotating cavity plays the role of $\mathcal{L}_{d}$ in the double-lid-driven flow (see also Sec. IV B 2). The robustness of this phenomenon has been explored by including an inner cylinder in the rotating cavity. ${ }^{65,66}$ Exploitation of this analogy may deepen insights into the onset of oscillatory regimes with further increasing nonlinearities in the present cavity flows.

\section{CONCLUSIONS}

This study presents a comparative numerical-experimental investigation of the global streamline patterns ("Lagrangian flow topologies") of three-dimensional (3D) flows in cavities under laminar and steady conditions. The main objective is to examine topological equivalences between flow classes and generic transport phenomena. To this end, two prototypical configurations are considered, buoyancy-driven and lid-driven flows.

A differentially heated cubical cavity with the direction of the temperature gradient perpendicular to gravity serves as the buoyancy-driven configuration. The lid-driven counterpart consists of a flow generated by the motion of two facing walls at the same speed in the opposite direction in a cylindrical cavity (double-liddriven flow). These systems are governed by the nondimensional Grashof (Gr) and Reynolds (Re) numbers, respectively.

Previous research established that fluid inertia prevails for small Gr and the buoyancy-driven flow exhibits a behavior that is analogous to single-lid-driven flows (motion of one endwall only). A buoyancy-induced bifurcation occurs for larger Gr, resulting in a flow topology that is reminiscent of double-lid-driven flows. ${ }^{22}$ This is the starting point of our investigation that concentrates on the analysis of the buoyancy-driven flow for larger Prandtl (Pr) and its equivalence with the double-lid-driven flow.

Two main symmetries organize the Lagrangian dynamics: (i) reflectional symmetry about the midplane and (ii) centrosymmetry about the central transverse line. These symmetries imply fundamental similarities between the streamline patterns of the considered cavity flows and enable a detailed study of generic phenomena based on two aspects: (i) dynamics in the symmetry plane and (ii) $3 \mathrm{D}$ dynamics outside the symmetry plane.

Bifurcations of the streamline topologies occur with increasing nonlinearities in the symmetry plane of the considered cavity flows. The Poincaré-Bendixson theorem governs the dynamics in this plane. In particular, the considered cavity flows are characterized by the same foci-saddle structure and a one-to-one correspondence between flows is possible in this case. This extends 
the above-mentioned link between the buoyancy-driven flow and single-lid-driven flows.

Moreover, the 3D flow topology is characterized by the coexistence of two families of KAM tori. Secondary toroidal structures appear with increasing nonlinearities. Furthermore, similarities with vortex breakdown flows regarding this structure are noted. The response of the flow topology to nonlinear perturbations is governed by universal Hamiltonian mechanisms.

Laboratory experiments validate several key aspects of the Lagrangian dynamics. Particle image velocimetry (PIV) measurements are considered to study the dynamics in the symmetry plane of the buoyancy-driven flow. Experimental analysis of tracer motion is performed by $3 \mathrm{D}$ particle-tracking velocimetry (PTV) in the double-lid-driven flow. PIV experiments show a good agreement between numerical and experimental bifurcations with increasing Gr. In particular, these experiments confirm the predicted bifurcation threshold for larger Pr. Moreover, a comparison between experimental trajectories obtained by PTV and their simulated counterparts reveals a good agreement with increasing Re. Taken together, the findings of this study support the universal character of the key aspects of the Lagrangian dynamics.

Future experimental efforts should focus on the identification of structures outside the symmetry plane (involving long-term experiments). Direct measurement of the two families of tori using PTV could be performed by using modified versions of the experimental lid-driven setup for higher Re (possibly using the alternative of moving bands instead of the finite-size walls).

Further research should be undertaken to explore the influence of the spatial Lagrangian structures on the time-dependent dynamics for further increasing nonlinearities. Symmetry breaking and full exploration of stagnation points and their interactions will be fundamental (also considering different cavity aspect ratios). In this context, reconciliation between the Lagrangian dynamics of the present flows and other configurations (especially vortex breakdown flows and Rayleigh-Bénard systems) may contribute to the understanding of unsteady cavity flows in general. Moreover, future studies might explore the important extension to nonpassive scalar transport and finite-size particles. ${ }^{1-3,18,67}$

\section{ACKNOWLEDGMENTS}

We sincerely acknowledge A. P. C. Holten, F. M. R. van Uittert, J. M. van der Veen, and G. W. J. M. Oerlemans for their technical assistance. P.S.C. gratefully acknowledges financial support from the Consejo Nacional de Ciencia y Tecnología (CONACYT), Mexico.

\section{APPENDIX: DOUBLE-LID-DRIVEN FLOW: PERFORMANCE OF THE TRACKING ALGORITHM}

A comparative analysis of particle tracking with the TaylorGalerkin scheme using the COMSOL velocity field for the double-liddriven flow is carried out in this section. Following Secs. IV C 1 and IV C 3, the Lagrangian dynamics in the symmetry plane and outside this plane are considered.

The first presented test concerns the limit $\mathrm{Re}=0$ in the symmetry plane $\mathcal{P}_{d}$. Figure 13(a) shows the comparison between the streamline patterns obtained using the tracking algorithm with the COMSOL field (red) and calculated by numerical simulation with the semianalytical solution in the noninertial limit according to Refs. 23 and 24 (black). The computed streamlines closely shadow each other. In full agreement with symmetries (7) and (8), closed orbits are obtained. Typical examples tracked for 50 revolutions using the COMSOL field are displayed in Fig. 13(b). Figure 13(c) presents the drift $\mathrm{X}_{n}=\left|x_{n}-x_{0}\right|$ of the intersection $x_{n}$ of each streamline with the $x$-axis after $n$ revolutions about the cavity center. Initial tracer positions $\left(x_{0}, z_{0}=0\right)$ are shown in Fig. 13(b) (markers). As exhibited in Fig. 13(c), streamlines are closed to an acceptable degree of precision, $\mathrm{X}_{n}<\mathcal{O}\left(10^{-2}\right)$.

Figure 14 shows the comparison between the streamline patterns in the symmetry plane for $\mathrm{Re}=500$ obtained using the COMSOL field and increased resolution. The computational mesh consists of $\mathcal{O}\left(3 \times 10^{5}\right)$ and $\mathcal{O}\left(10^{6}\right)$ elements in figures Figs. 14(a) and 14(b), respectively. Three tracer trajectories are displayed (distinguished by color). The flow topology remains unchanged (variations are entirely quantitative).

A comparison regarding the $3 \mathrm{D}$ dynamics is shown in Fig. 15 by exposing the Poincaré section of typical tracer trajectories (distinguished by color) for $\mathrm{Re}=200$ using the COMSOL field and increased (a)

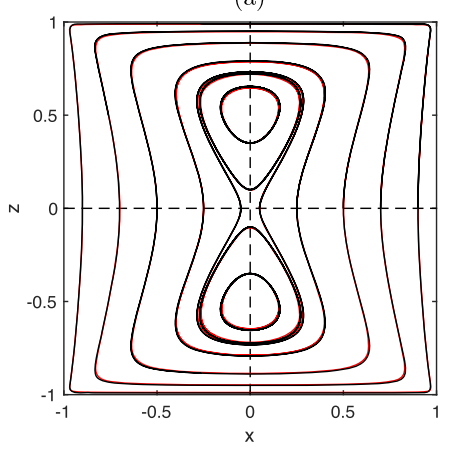

(b)

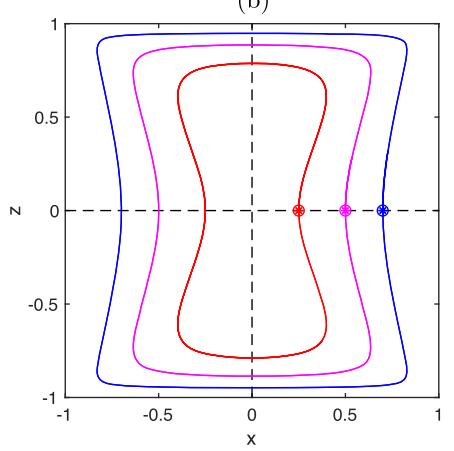

(c)

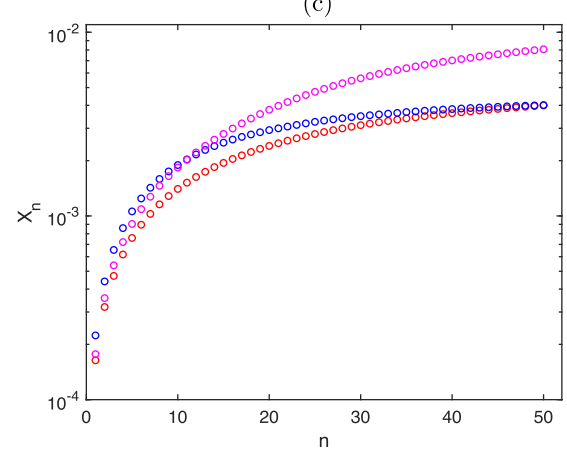

FIG. 13. Double-lid-driven flow, $\mathrm{Re}=0$. Streamlines in the symmetry plane $(y=0)$. (a) Comparison between the streamline pattern; semianalytical solution (black), COMSOL field (red, $\mathcal{O}\left(10^{6}\right)$ mesh elements). (b) Typical streamlines obtained using the COMSOL field, initial tracer positions (markers). (c) Drift $X_{n}$ corresponding to intersections of the streamlines in (b) with the positive $x$-axis as a function of the number of revolutions $n$. 
(a)

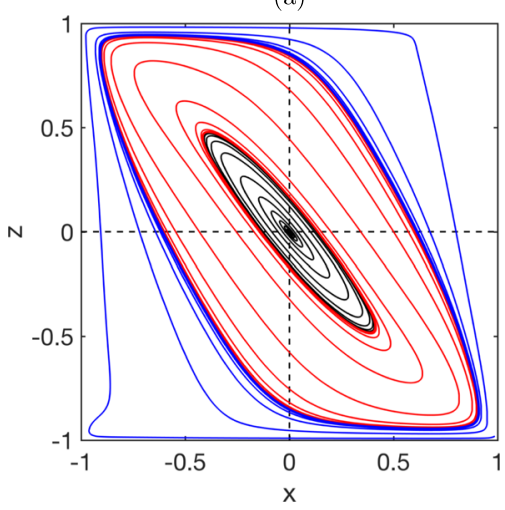

(b)

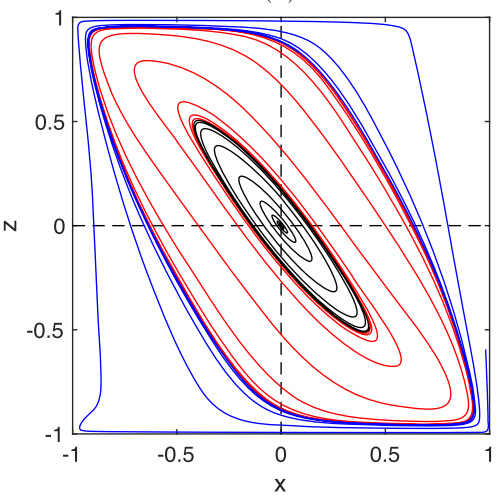

FIG. 14. Double-lid-driven flow, $\operatorname{Re}=500$. Streamline pattern in the symmetry plane $(y=0)$ obtained with the COMSOL field and increased resolution. (a) $\mathcal{O}\left(3 \times 10^{5}\right)$ mesh elements. (b) $\mathcal{O}\left(10^{6}\right)$ mesh elements. (a)
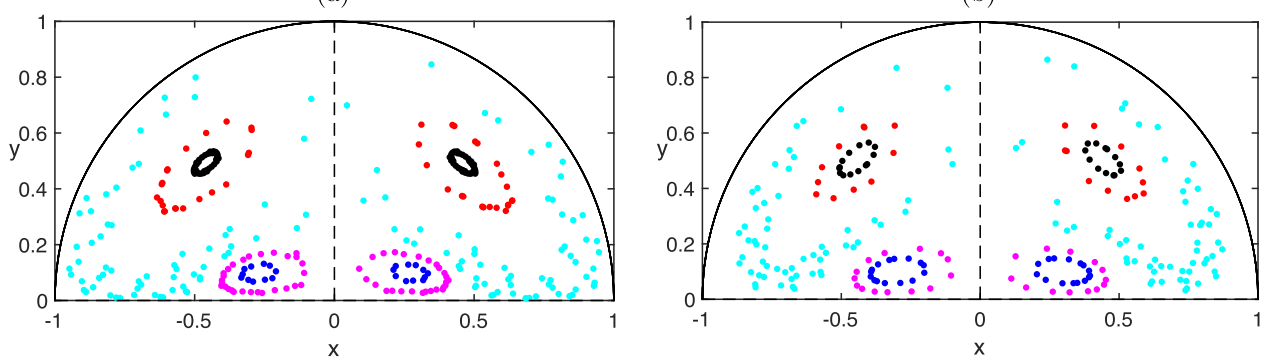

FIG. 15. Double-lid-driven flow, $R e$ $=200$. Poincaré section $(z=0)$ obtained with the COMSOL field and increased resolution. (a) $\mathcal{O}\left(3 \times 10^{5}\right)$ mesh elements. (b) $\mathcal{O}\left(10^{6}\right)$ mesh elements. resolution. The coexistence of two families of KAM tori is displayed, and differences are, again, entirely quantitative. Similar tests for different Re confirm the robustness of the simulated Lagrangian dynamics. This indicates an adequate degree of resolution.

\section{REFERENCES}

${ }^{1}$ H. Aref, J. R. Blake, M. Budišić, S. S. S. Cardoso, J. H. E. Cartwright, H. J. H. Clercx, K. El Omari, U. Feudel, R. Golestanian, E. Gouillart, G. F. van Heijst, T. S. Krasnopolskaya, Y. Le Guer, R. S. MacKay, V. V. Meleshko, G. Metcalfe, I. Mezić, A. P. S. de Moura, O. Piro, M. F. M. Speetjens, R. Sturman, J.-L. Thiffeault, and I. Tuval, "Frontiers of chaotic advection," Rev. Mod. Phys. 89, 025007 (2017)

${ }^{2}$ M. F. M. Speetjens, G. Metcalfe, and M. Rudman, "Lagrangian transport and chaotic advection in three-dimensional laminar flows," preprint arXiv: 1904.07580 (2019).

${ }^{3}$ H. Aref, "Chaotic advection of fluid particles," Philos. Trans. R. Soc., A 333, 273-288 (1990).

${ }^{4}$ E. Ott, Chaos in Dynamical Systems (Cambridge University Press, 2002).

${ }^{5}$ P. G. Bakker, Bifurcations in Flow Patterns (Kluwer Academic Publishers, 1991).

${ }^{6}$ R. S. MacKay, "Transport in 3D volume-preserving flows," J. Nonlinear Sci. 4, 329 (1994).

${ }^{7} \mathrm{M}$. Brøns, "Streamline topology: Patterns in fluid flows and their bifurcations," Adv. Appl. Mech. 41, 1-42 (2007).

${ }^{8}$ M. F. M. Speetjens, E. A. Demissie, G. Metcalfe, and H. J. H. Clercx, "Lagrangian transport characteristics of a class of three-dimensional inline-mixing flows with fluid inertia," Phys. Fluids 26, 113601 (2014).

${ }^{9}$ O. Baskan, H. Rajaei, M. F. M. Speetjens, and H. J. H. Clercx, "Scalar transport in inline mixers with spatially periodic flows," Phys. Fluids 29, 013601 (2017).

${ }^{10}$ W. Arter, "Ergodic stream-lines in steady convection," Phys. Lett. A 97, 171-174 (1983).
${ }^{11}$ W. Arter, "Nonlinear Rayleigh-Bénard convection with square planform," J. Fluid Mech. 152, 391-418 (1985).

${ }^{12}$ A. A. Chernikov and G. Schmidt, "Chaotic streamlines in convective cells," Phys. Lett. A 169, 51-56 (1992).

${ }^{13} \mathrm{~J}$. M. Ottino, The Kinematics of Mixing: Stretching, Chaos, and Transport (Cambridge University Press, 1989).

${ }^{14}$ P. N. Shankar and M. D. Deshpande, "Fluid mechanics in the driven cavity," Annu. Rev. Fluid Mech. 32, 93-136 (2000).

${ }^{15} \mathrm{P}$. N. Shankar, Slow Viscous Flows: Qualitative Features and Quantitative Analysis Using Complex Eigenfunction Expansions (Imperial College Press, 2007).

${ }^{16}$ C. Simó, D. Puigjaner, J. Herrero, and F. Giralt, "Dynamics of particle trajectories in a Rayleigh-Bénard problem," Commun. Nonlinear Sci. Numer. Simul. 15, 24-39 (2010).

${ }^{17}$ M. Lappa, Thermal Convection: Patterns, Evolution and Stability (John Wiley \& Sons, 2010).

${ }^{18}$ H. C. Kuhlmann and F. Romanò, "The lid-driven cavity," in Computational Modelling of Bifurcations and Instabilities in Fluid Dynamics, edited by A. Gelfgat (Springer, 2019), pp. 233-309.

${ }^{19}$ W. J. Hiller, S. Koch, and T. A. Kowalewski, "Three-dimensional structures in laminar natural convection in a cubic enclosure," Exp. Therm. Fluid Sci. 2, 34-44 (1989).

${ }^{20}$ T. W. H. Sheu and S. F. Tsai, "Flow topology in a steady three-dimensional liddriven cavity," Comput. Fluids 31, 911-934 (2002).

${ }^{21}$ A. Povitsky, "Three-dimensional flow with elevated helicity in driven cavity by parallel walls moving in perpendicular directions," Phys. Fluids 29, 083601 (2017).

${ }^{22}$ P. S. Contreras, M. F. M. Speetjens, and H. J. H. Clercx, "Lagrangian transport in a class of three-dimensional buoyancy-driven flows," J. Fluid Mech. 832, 5-40 (2017).

${ }^{23}$ P. N. Shankar, "Three-dimensional eddy structure in a cylindrical container," J. Fluid Mech. 342, 97-118 (1997).

${ }^{24}$ P. N. Shankar, "Three-dimensional Stokes flow in a cylindrical container," Phys. Fluids 10, 540-549 (1998). 
${ }^{25} \mathrm{~T}$. W.-H. Sheu and R.-K. Lin, "Three-dimensional bifurcations in a cubic cavity due to buoyancy-driven natural convection," Int. J. Heat Mass Transfer 54, 447467 (2011).

${ }^{26}$ R.-K. Lin and W.-H. Sheu, "Study of cellular flow structure and pitchfork bifurcation in a laterally-heated cube," Int. J. Heat Mass Transfer 83, 39-50 (2015).

${ }^{27}$ A. Medelfef, D. Henry, A. Bouabdallah, and S. Kaddeche, "Bifurcations from steady to quasi-periodic flows in a laterally heated cavity filled with low Prandtl number fluids," J. Fluid Mech. 861, 223-252 (2019).

${ }^{28}$ I. Mercader, O. Sánchez, and O. Batiste, "Secondary flows in a laterally heated horizontal cylinder," Phys. Fluids 26, 014104 (2014).

${ }^{29}$ M. R. Ravi, R. A. W. M. Henkes, and C. J. Hoogendoorn, "On the highRayleigh-number structure of steady laminar natural-convection flow in a square enclosure," J. Fluid Mech. 262, 325-351 (1994).

${ }^{30}$ R. J. A. Janssen and R. A. W. M. Henkes, "Influence of Prandtl number on instability mechanisms and transition in a differentially heated square cavity," J. Fluid Mech. 290, 319-344 (1995).

${ }^{31}$ R. J. A. Janssen, R. A. W. M. Henkes, and C. J. Hoogendoorn, "Transition to time-periodicity of a natural-convection flow in a 3D differentially heated cavity," Int. J. Heat Mass Transfer 36, 2927-2940 (1993).

${ }^{32}$ T. Fusegi and J. M. Hyun, "Laminar and transitional natural convection in an enclosure with complex and realistic conditions," Int. J. Heat Fluid Flow 15, 258-268 (1994).

${ }^{33}$ S. J. M. Linthorst, W. M. M. Schinkel, and C. J. Hoogendoorn, "Flow structure with natural convection in inclined air-filled enclosures," J. Heat Transfer 103, 535-539 (1981).

${ }^{34}$ W. J. Hiller, S. Koch, T. A. Kowalewski, G. de Vahl Davis, and M. Behnia, "Experimental and numerical investigation of natural convection in a cube with two heated side walls," in Proceedings IUTAM Symposium, edited by H. K. Moffatt and A. Tsinober (Cambridge University Press, 1990), pp. 717-726.

${ }^{35}$ L. Soucasse, P. Rivière, A. Soufiani, S. Xin, and P. Le Quéré, "Transitional regimes of natural convection in a differentially heated cubical cavity under the effects of wall and molecular gas radiation," Phys. Fluids 26, 024105 (2014).

${ }^{36}$ S. Bouarab, F. Mokhtari, S. Kaddeche, D. Henry, V. Botton, and A. Medelfef, "Theoretical and numerical study on high frequency vibrational convection: Influence of the vibration direction on the flow structure," Phys. Fluids 31, 043605 (2019).

${ }^{37} \mathrm{O}$. Shishkina and S. Horn, "Thermal convection in inclined cylindrical containers," J. Fluid Mech. 790, R3 (2016).

${ }^{38} \mathrm{Q}$. Wang, Z.-H. Wan, R. Yan, and D.-J. Sun, "Flow organization and heat transfer in two-dimensional tilted convection with aspect ratio 0.5 ," Phys. Fluids $\mathbf{3 1}$, 025102 (2019).

${ }^{39}$ P. S. Contreras, L. M. de la Cruz, and E. Ramos, "Topological analysis of a mixing flow generated by natural convection," Phys. Fluids 28, 013602 (2016).

${ }^{40}$ Y. Liu, Y. Bian, Y. Zhao, S. Zhang, and Q. Suo, "Scaling laws for the transient convective flow in a differentially and linearly heated rectangular cavity at $\operatorname{Pr}>1$," Phys. Fluids 31, 043601 (2019).

${ }^{41} \mathrm{~F}$. Gürcan, "Effect of the Reynolds number on streamline bifurcations in a double-lid-driven cavity with free surfaces," Comput. Fluids 32, 1283-1298 (2003).

${ }^{42}$ F. Romanò, S. Albensoeder, and H. C. Kuhlmann, "Topology of threedimensional steady cellular flow in a two-sided anti-parallel lid-driven cavity," J. Fluid Mech. 826, 302-334 (2017).

${ }^{43}$ H. C. Kuhlmann, M. Wanschura, and H. J. Rath, "Flow in two-sided lid-driven cavities: Non-uniqueness, instabilities, and cellular structures," J. Fluid Mech. 336, 267-299 (1997).

${ }^{44} \mathrm{C}$. Blohm and H. C. Kuhlmann, "The two-sided lid-driven cavity: Experiments on stationary and time-dependent flows," J. Fluid Mech. 450, 67-95 (2002).
${ }^{45}$ T. Siegmann-Hegerfeld, S. Albensoeder, and H. C. Kuhlmann, "Two-and threedimensional flows in nearly rectangular cavities driven by collinear motion of two facing walls," Exp. Fluids 45, 781-796 (2008).

${ }^{46}$ I. Ataei-Dadavi, M. Chakkingal, S. Kenjeres, C. R. Kleijn, and M. J. Tummers, "Flow and heat transfer measurements in natural convection in coarse-grained porous media," Int. J. Heat Mass Transfer 130, 575-584 (2019).

${ }^{47}$ I. Ataei-Dadavi, N. Rounaghi, M. Chakkingal, S. Kenjeres, C. R. Kleijn, and M. J. Tummers, "An experimental study of flow and heat transfer in a differentially side heated cavity filled with coarse porous media," Int. J. Heat Mass Transfer 143, 118591 (2019).

${ }^{48}$ M. F. M. Speetjens, H. J. H. Clercx, and G. J. F. van Heijst, "A numerical and experimental study on advection in three-dimensional Stokes flows," J. Fluid Mech. 514, 77-105 (2004).

${ }^{49} \mathrm{~J}$. Willneff, "A spatio-temporal matching algorithm for 3D particle tracking velocimetry," Ph.D. thesis, ETH, Zürich, Switzerland, 2003.

${ }^{50}$ J. Znaien, M. F. M. Speetjens, R. R. Trieling, and H. J. H. Clercx, "Observability of periodic lines in three-dimensional lid-driven cylindrical cavity flows," Phys. Rev. E 85, 066320 (2012).

${ }^{51}$ M. F. M. Speetjens, H. J. H. Clercx, and G. J. F. van Heijst, "Merger of coherent structures in time-periodic viscous flows," Chaos 16, 043104 (2006).

${ }^{52}$ G. de Vahl Davis, "Mostly natural convection," in 5th European ThermalSciences Conference, Eindhoven, The Netherlands, 2008.

${ }^{53}$ S. Wiggins, Introduction to Applied Nonlinear Dynamical Systems and Chaos (Springer, 2003).

${ }^{54}$ U. Dallmann, "Structural stability of three-dimensional vortex flows," in Nonlinear Dynamics of Transcritical Flows, edited by H. L. Jordan, H. Oertel, and K. Robert (Springer, 1985), pp. 81-102.

${ }^{55} \mathrm{R}$. Kessler, "Nonlinear transition in three-dimensional convection," J. Fluid Mech. 174, 357-379 (1987).

${ }^{56}$ A. Ern and M. D. Smooke, "Vorticity-velocity formulation for threedimensional steady compressible flows," J. Comput. Phys. 105, 58-71 (1993).

${ }^{57}$ W. J. Hiller, S. Koch, T. A. Kowalewski, P. Mitgau, and K. Range, "Visualization of 3-D natural convection," in Flow Visualization VI, edited by Y. Tanida and H. Miyashiro (Springer, 1992), pp. 474-478.

${ }^{58}$ A. L. Yarin, T. A. Kowalewski, W. J. Hiller, and St. Koch, "Distribution of particles suspended in convective flow in differentially heated cavity," Phys. Fluids 8 , 1130-1140 (1996).

${ }^{59}$ A. Y. Gelfgat, P. Z. Bar-Yoseph, and A. Solan, "Steady states and oscillatory instability of swirling flow in a cylinder with rotating top and bottom," Phys. Fluids 8, 2614-2625 (1996).

${ }^{60}$ A. Y. Gelfgat, P. Z. Bar-Yoseph, and A. Solan, "Stability of confined swirling flow with and without vortex breakdown," J. Fluid Mech. 311, 1-36 (1996).

${ }^{61}$ B. R. Munson and D. D. Joseph, "Viscous incompressible flow between concentric rotating spheres. Part 1. Basic flow," J. Fluid Mech. 49, 289-303 (1971).

${ }^{62}$ K. Bajer and H. K. Moffatt, "Chaos associated with fluid inertia," in Topological Aspects of the Dynamics of Fluids and Plasmas, edited by H. K. Moffatt, G. M. Zaslavsky, M. Tabor, and P. Comte (Springer, 1992), pp. 517-534.

${ }^{63}$ J. H. E. Cartwright, M. Feingold, and O. Piro, "Chaotic advection in threedimensional unsteady incompressible laminar flow," J. Fluid Mech. 316, 259-284 (1996).

${ }^{64} \mathrm{~J}$. Guckenheimer and P. Holmes, Nonlinear Oscillations, Dynamical Systems, and Bifurcations of Vector Fields (Springer, 1983), Vol. 42.

${ }^{65} \mathrm{~T}$. Mullin, S. J. Tavener, and K. A. Cliffe, "On the creation of stagnation points in a rotating flow," J. Fluids Eng. 120, 685-689 (1998).

${ }^{66}$ R. E. Hewitt, T. Mullin, S. J. Tavener, M. A. I. Khan, and P. D. Treacher, "Nonlinear vortex development in rotating flows," Philos. Trans. R. Soc., A 366, 1317-1329 (2007).

${ }^{67}$ F. Romanò, P. K. Kannan, and H. C. Kuhlmann, "Finite-size Lagrangian coherent structures in a two-sided lid-driven cavity," Phys. Rev. Fluids 4, 024302 (2019). 